\title{
Boundedness of Convex Polytopes Networks via Local Fractional Metric Dimension
}

\author{
Muhammad Javaid (D, ${ }^{1}$ Hassan Zafar, ${ }^{1}$ Amer Aljaedi, ${ }^{2}$ \\ and Abdulaziz Mohammad Alanazi iD $^{3}$ \\ ${ }^{1}$ Department of Mathematics, School of Science, University of Management and Technology, Lahore 54770, Pakistan \\ ${ }^{2}$ College of Computing and Information Technology, University of Tabuk, Tabuk, Saudi Arabia \\ ${ }^{3}$ Department of Mathematics, University of Tabuk, Tabuk, Saudi Arabia
}

Correspondence should be addressed to Muhammad Javaid; javaidmath@gmail.com

Received 13 October 2021; Accepted 23 November 2021; Published 15 December 2021

Academic Editor: Muhammad Faisal Nadeem

Copyright (c) 2021 Muhammad Javaid et al. This is an open access article distributed under the Creative Commons Attribution License, which permits unrestricted use, distribution, and reproduction in any medium, provided the original work is properly cited.

\begin{abstract}
Metric dimension is one of the distance-based parameter which is frequently used to study the structural and chemical properties of the different networks in the various fields of computer science and chemistry such as image processing, pattern recognition, navigation, integer programming, optimal transportation models, and drugs discovery. In particular, it is used to find the locations of robots with respect to shortest distance among the destinations, minimum consumption of time, and lesser number of the utilized nodes and to characterize the chemical compounds having unique presentation in molecular networks. The fractional metric dimension being a latest developed weighted version of the metric dimension is used in the distance-related problems of the aforementioned fields to find their nonintegral optimal solutions. In this paper, we have formulated the local resolving neighborhoods with their cardinalities for all the edges of the convex polytopes networks to compute their local fractional metric dimensions in the form of exact values and sharp bounds. Moreover, the boundedness of all the obtained results is also proved.
\end{abstract}

\section{Introduction}

There are various important tools in the subject of graph theory that are used to model real life problems such as school bus routing, networks of communication, allocation of the frequencies to the radio stations, and social networks consisting of establishing and working relationships between people. Slater formally defined the concept of resolving (locating) sets for a connected network and designed an algorithm to compute them [1]. Harary and Melter also studied the resolving sets of a connected network, and they called the minimum cardinality of a resolving set as metric dimension (MD) [2]. Gary and Johnson proved that the formulating of MD in its general form for all the connected networks is an NP complete problem that leads to characterize the certain families of networks with fixed MD [3]. Tomescu and Melter applied a metric basis in digital geometry and derived some fundamental results about the existence of the rectangles having minimal metric bases of any size greater or equal to three [4].

Chartrand et al. [5] used MD to solve integer programming problem (IPP) with specific conditions. They defined independence resolvent set and established the metric independence number for different families of connected networks. In particular, they also established bounds of the MD of unicyclic networks and proved that MD of a connected network $\mathbb{G}$ is 1 iff $\mathbb{G} \cong P_{n}$, where $P_{n}$ (path). Metric dimension generalized Peterson multinetworks $(P(2 n, n))$ are studied in [6], and bounded metric dimension of generalized Peterson network is computed in [7]. After that, local MD [8], strong MD [9], $k$-metric dimension [10], and sharp bounds of partition dimension of convex polytopes are studied [11] in the form of different mathematical expressions and computing algorithms. Moreover, constant MD of some generalized convex polytopes is studied by Zuo et al. [12], and unbounded MD of 
splitting networks $\left(S\left(\mathbb{P}_{n}\right), S\left(\mathbb{C}_{n}\right)\right)$ of a path and cycle is computed by Pan et al. [13]. For further study of MD and its applications, we refer [14-16].

The fractional version of MD known as fractional metric dimension (FMD) is introduced by Currie and Oellermann, and they found the nonintegral solutions of IPP by using it [17]. Fahar et al. proposed the optimal solution of linear relaxation of the IPP by using FMD [18]. Arguman and Mathew formally defined the concept of FMD and introduced many basic properties of FMD about the certain families of the connected networks [19]. The bounds of FMD of all the connected networks are established by Alkhalidi et al. [20]. In particular, the FMDs of generalized Jahangir, trees, and unicyclic networks are computed in [21, 22]. Recently, Javaid et al. [23] characterized all the networks with FMD exactly one. The bounds of FMD of metal organic networks and cartesian product of connected networks are computed in [24, 25].

Aisyah et al. [26] introduced the idea of local fractional metric dimension (LFMD), and they also calculated the exact values of LFMD for corona product of connected networks. For all the connected networks, Javaid et al. [27] established upper bound of LFMD. Furthermore, Javaid et al. [28] improved the lower bound of LFMD from unity and illustrated this result by computing the LFMD for the prism-related networks.

Now, we present applications of metric-based dimensions in different fields. In chemistry, the various structures of chemical compounds are considered as the sets of functional groups, where atoms and bonds are presented by vertices and edges, respectively. By permuting the positions of the sets of functional groups, different common substructures are characterized. The concept of resolving sets determines the particular position when two compounds share the same functional groups. This investigation plays a supreme role in drug discovery and pharmaceutical activities. On the other hand, MD is an essential tool to operate movement of navigating agents (reboots) in large sets of landmarks where the resolving sets uniquely determine the positions of navigating agents in the graph space. The problem of minimum machines (robots) to be placed at certain nodes to trace each and every node exactly once is worth investigating in sonar and loran stations. For more details, we refer to [3, 29].

In this paper, we computed LFMD of some families of convex polyposes networks in the form of exact values and sharp bounds. It is also obtained that in all the cases, LFMD of convex polytopes remains bounded. The remaining manuscript includes Sections 2-4 consisting of preliminaries, main results, and conclusion, respectively.

\section{Preliminaries}

Let $\mathbb{G}=(V(\mathbb{G}), E(\mathbb{G}))$ be a network with $V(\mathbb{G})$ as vertex-set and $E(\mathbb{G}) \subseteq V(\mathbb{G}) \times V(\mathbb{G})$ as edge-set. A path is a simple network in which vertices can be ordered such as two vertices are adjacent if and only of they are consecutive along the list. A network $\mathbb{G}$ is connected if each pair of vertices of $\mathbb{G}$ leads a path. For any two vertices $\{u, v\} \subseteq V(\mathbb{G})$, the distance $(d(u, v))$ is the number of edges in a shortest path between them. For further study of preliminary concepts of the subject graph theory, see [30].

For a connected network $\mathbb{G}$, a vertex $x \in V(\mathbb{G})$ resolves $\{u, v\} \subseteq V(\mathbb{G})$ if $d(x, u) \neq d(x, v)$. Let $U=\left\{u_{1}, u_{2}, u_{3}, \ldots\right.$, $\left.u_{m}\right\} \subseteq V(\mathbb{G})$ and $u \in V(\mathbb{G})$, then $m$-tuple representation of $u$ with respect to $U$ donated by $r(u \mid U)$ is defined as $r(u \mid U)=\left(r\left(u, u_{1}\right), r\left(u, u_{2}\right), r\left(u, u_{3}\right), \ldots, r\left(u, u_{m}\right)\right)$. If the distinct vertices of $\mathbb{G}$ have different representations with respect to $U$, then $U$ is called a resolving set of $\mathbb{G}$. The resolving sets of minimum cardinalities are called metric bases in $\mathbb{G}$, and the cardinality of a metric basis is known as metric dimension (MD) of $\mathbb{G}$ that is defined as follows:

$$
\operatorname{dim}(\mathbb{G})=\min \{|U|: U \text { is are solving set of } \mathbb{G}\}[2]
$$

For each pair of two adjacent vertices $u, v \in V(\mathbb{G})$, the local resolving neighborhood (LRN) set is defined as

$$
\operatorname{LR}(u v)=\{a \in V(\mathbb{G}): d(a, u) \neq d(a, v)\} .
$$

A local resolving function (LRF) is a real-valued function $\mathrm{F}: V(\mathbb{G}) \longrightarrow[0,1]$ such that $\mathrm{F}(\operatorname{LR}(u v)) \geq 1$ for each $\operatorname{LR}(u v)$ of $\mathbb{G}$, where $\mathrm{F}(\mathrm{LR}(u v))=\sum_{x \in \operatorname{LR}(u v)} \mathrm{F}(x)$. A LRF $\mathrm{F}$ of $\mathbb{G}$ is called minimal if there exists some other function $\mathrm{F}^{\prime}: V(\mathbb{G}) \longrightarrow[0,1]$ such that $\mathrm{F}^{\prime} \leq \delta$ and $\delta(k) \neq \mathrm{F}(k)$ for at least one $u \in V(\mathbb{G})$ that is not LRF of $\mathbb{G}$. If $F$ presents all the minimal resolving functions of $\mathbb{G}$, then its LFMD is defined as $\operatorname{dim}_{l f}(\mathbb{G})=\min \{|F|\}$, where $|F|=\sum_{u \in V(\mathbb{G})} F(u)[26]$.

The networks of convex polytopes are fundamental geometric objects that have been investigated since antiquity. They are playing an important role in mathematical subjects like algebraic geometry, combinatorial optimization, and graph theory. For a connected network $\mathbb{G}$, Baca et al. [29] constructed the convex polytopes network $R_{m}$ by the combination of prism and antiprism networks, where $m \geq 5$. It consists of inner $\left(\left\{u_{i}: 1 \leq i \leq m\right\}\right)$, middle $\left(\left\{v_{i}: 1 \leq i \leq m\right\}\right)$, and outer $\left(\left\{w_{i}: 1 \leq i \leq m\right\}\right)$ cycle vertices with $\left|V\left(R_{m}\right)\right|=3 m$ and $E\left(R_{m}\right)=\left\{u_{i} u_{i+1}, v_{i} v_{i+1}, w_{i} w_{i+1}, u_{i} v_{i}\right.$, $\left.u_{i+1} v_{i}, v_{i} w_{i}: 1 \leq i \leq m-1, i+1 \cong(1 \bmod m)\right\}$. For more details, see Figure 1.

A network of convex polytope $A_{m}$ is obtained from $R_{m}$ by inserting new edges $v_{i} w_{i+1}$, where $1 \leq i \leq m-1$, $i+1 \cong(1 \bmod m)$, and $\left|V\left(A_{m}\right)\right|=3 m$ (see Figure 2$)$. A network of convex polytope $S_{m}$ is formed by connecting the alternating bands of triangles of two copies of prism networks. It consists of inner $\left(u_{i}\right)$, middle $\left(v_{i} \& w_{i}\right)$, and outer $\left(z_{i}\right)$ cycle vertices with $1 \leq i \leq m$ and $\left|V\left(S_{m}\right)\right|=4 m$. The edge-set of $S_{m}$ is defined as $E\left(S_{m}\right)=\left\{u_{i} u_{i+1}, v_{i} v_{i+1}, w_{i}\right.$ $w_{i+1}, z_{i} z_{i} \quad+1, u_{i} v_{i}, v_{i} w_{i}, v_{i+1} w_{i}, w_{i} z_{i}: 1 \leq i \leq m-1, i+1 \cong$ $(1 \bmod m)\}$. For more details, see Figure 3 . The network of convex polytope $Q_{m}$ is obtained from $S_{m}$ by deleting edges $w_{i} w_{i+1}$ as shown in Figure 4.

Now, we define some important results which will be frequently used in the main results as follows.

Theorem 1 (see [27]). For connected network $\mathbb{G}=(V(\mathbb{G}), E(\mathbb{G}))$ and the local resolving neighborhood $(L R N)$ set $L R(e)$ of the edge $e$, if $|L R(e) \cap A| \geq \gamma, \forall e \in E(\mathbb{G})$, then 


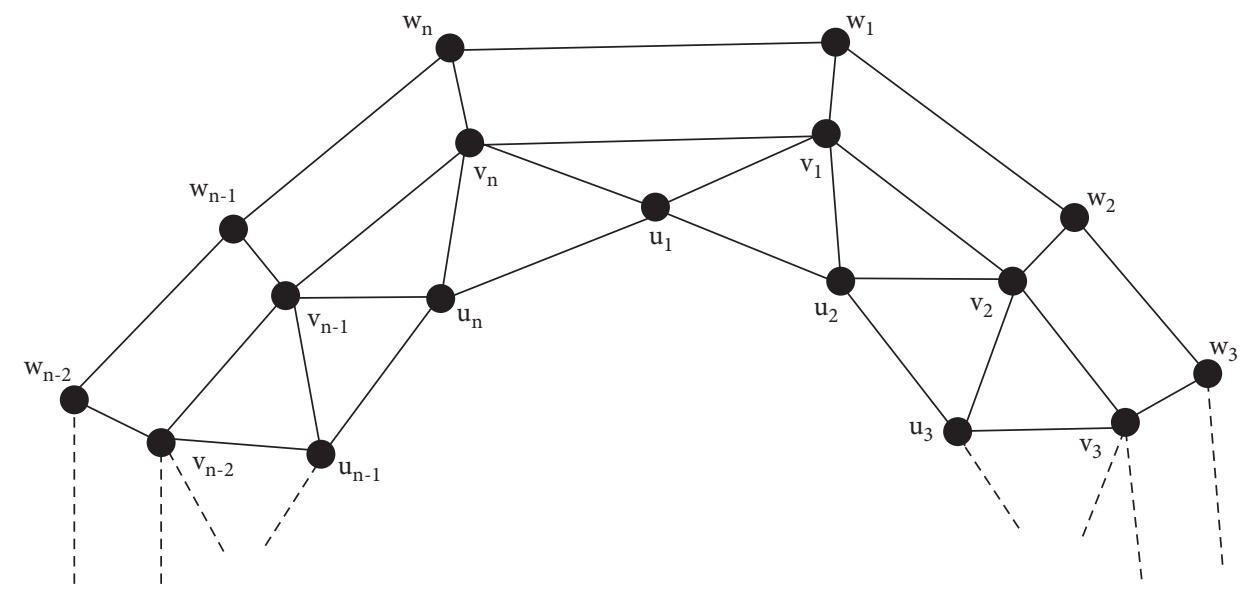

FIgURe 1: Convex polytope $R_{m}$.

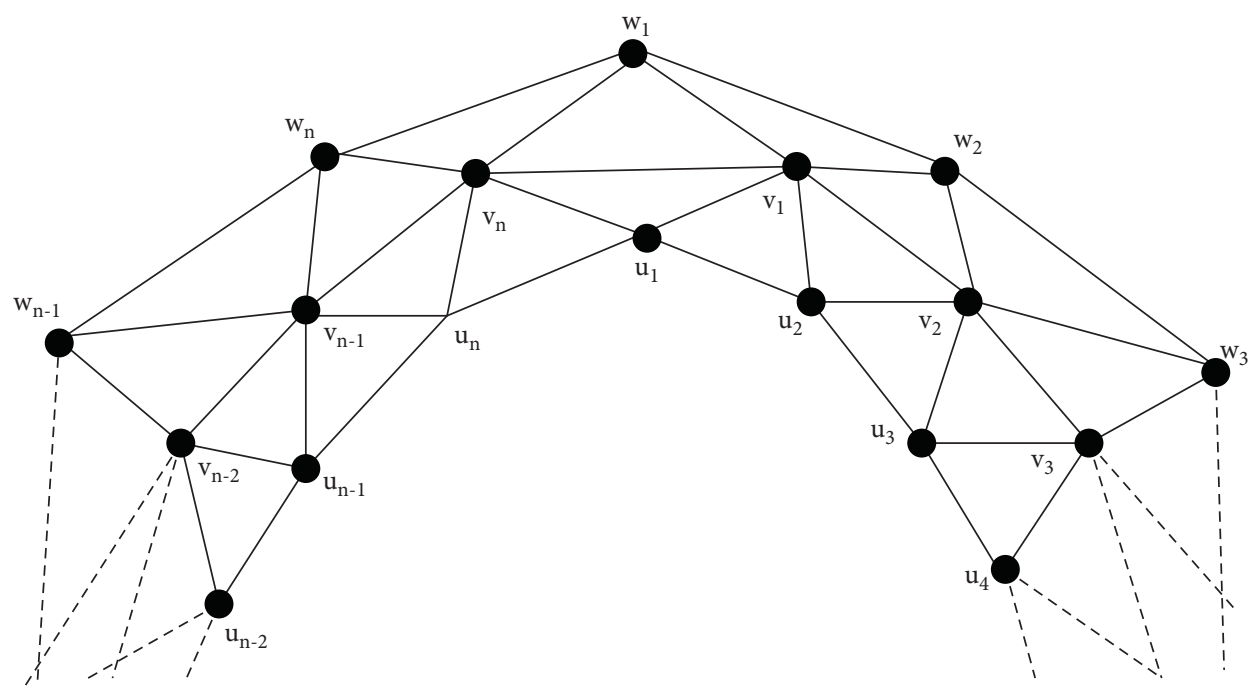

Figure 2: Convex polytope $A_{m}$.

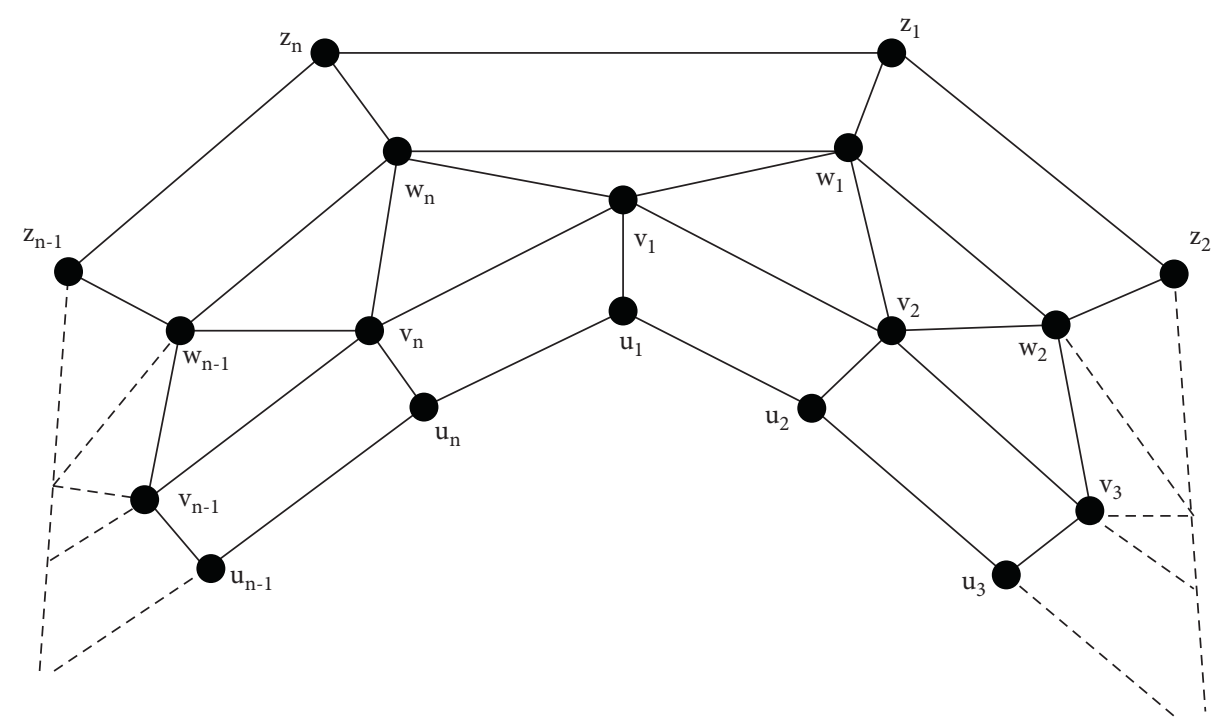

Figure 3: Convex polytope $S_{m}$. 


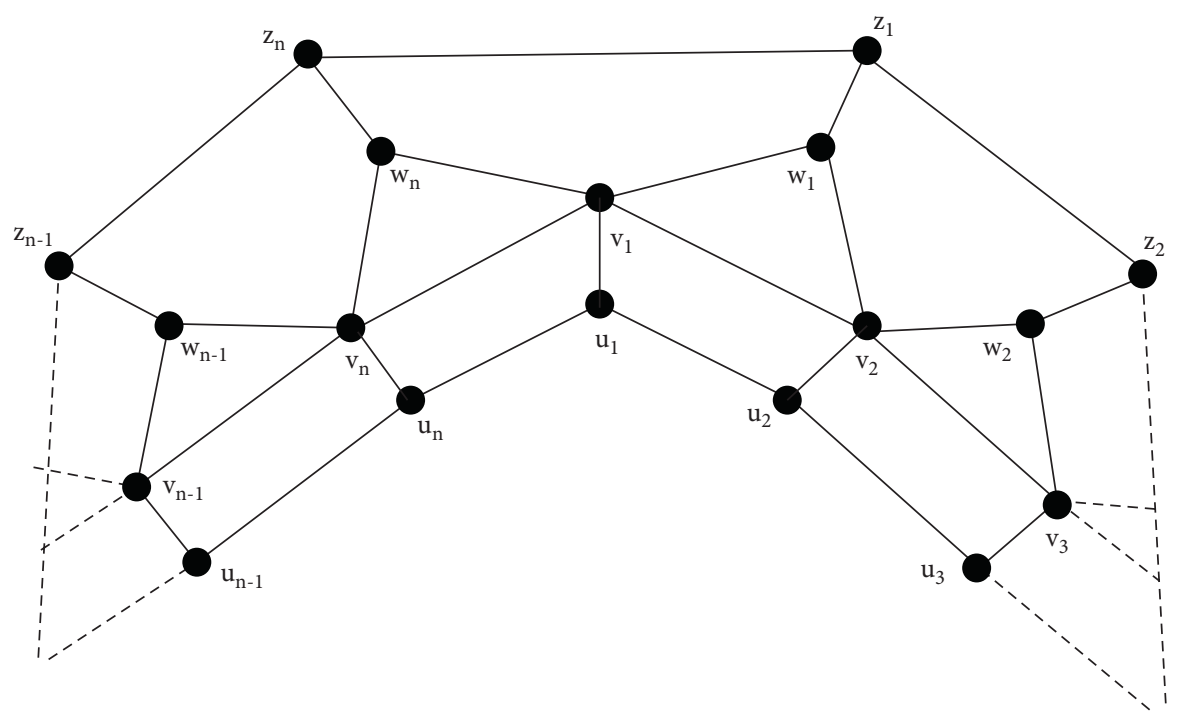

FIgURe 4: Convex polytope $Q_{m}$.

$$
1 \leq \operatorname{dim}_{l f}(\mathbb{G}) \leq \frac{|A|}{\gamma}
$$

where $2 \leq \gamma \leq|V(\mathbb{G})|, \quad \gamma=\min \{|L R(e)|: e \in E(\mathbb{G})\}$, and $A=\cup\{L R(e):|L R(e)|=\gamma\}$.

Theorem 2 (see $[28])$. Let $\mathbb{G}=(V(\mathbb{G}), E(\mathbb{G}))$ be a connected network with $V(\mathbb{G})$ be the vertex-set and $L R(e)$ be the LRN set for the edge e of $\mathbb{G}$. Then,

$$
\frac{|V(\mathbb{G})|}{\Gamma} \leq \operatorname{dim}_{l f}(\mathbb{G})
$$

where $2 \leq \Gamma \leq|V(\mathbb{G})|$ and $\Gamma=\max \{|L R(e)|: e \in E(\mathbb{G})\}$.

Corollary 1 (see [28]). Let $\mathbb{G}=(V(\mathbb{G}), E(\mathbb{G}))$ be a connected network and $L R(e)$ be $L R N$ of $e \in E(\mathbb{G})$. If $\gamma=\delta$ and $X=V(\mathbb{G})$, then

$$
\operatorname{dim}_{l f}(\mathbb{G})=\frac{|V(\mathbb{G})|}{\delta},
$$

$\delta=\max \{|L R(e)|: e \in E(\mathbb{G})\}, \quad \gamma=\min \{|L R(e)|: e \in E(\mathbb{G})\}$, and $X=\cup\{L R(e):|L R(e)| \gamma\}$.

\section{Main Results}

In this section, we investigate boundedness of LFMD of different convex polytopes networks.

3.1. LRN Sets and LFMD of Convex Polytope $R_{m}$. This subsection covers the results related to the LRN sets and LFMD of the convex polynope network that is presented by $R_{m}$ for any integral value $m \geq 5$.

Lemma 1. Let $R_{m}$ be a network of convex polytope, where $m \geq 5$ and $m \cong 1(\bmod 2)$. Then, for $1 \leq i \leq m$,

(a) $\left|\operatorname{LR}\left(e_{i}\right)\right|=\left|\operatorname{LR}\left(u_{i} v_{i}\right)\right|=\left|\operatorname{LR}\left(v_{i} u_{i+1}\right)\right|=((3 m+3) / 2)$, $\left|\operatorname{LR}\left(v_{i} w_{i}\right)\right|=3 m$, and $\left|\cup_{i=1}^{m} \operatorname{LR}\left(e_{i}\right)\right|=3 m$ (b) $\left|L R\left(e_{i}\right)\right| \leq|L R(x)| \leq\left|L R\left(v_{i} w_{i}\right)\right|, \forall x \in E\left(R_{m}\right)$

(c) $\left|L R(x) \cap \cup_{i=1}^{m} L R\left(e_{i}\right)\right| \geq\left|L R\left(e_{i}\right)\right|, \forall x \in E\left(R_{m}\right)$

Proof. Assume that $u_{i}, v_{i}$, and $w_{i}$ are inner, middle, and outer vertices of $R_{m}$, respectively, where $1 \leq i \leq m$ and $m+1 \cong 1(\bmod m)$.

(a) Since $\operatorname{LR}\left(u_{i} v_{i}\right)=\left\{u_{i}, u_{((m+2 i+1) / 2)}, u_{((m+2 i+3) / 2)}, \ldots\right.$, $u_{m}, v_{i}, v_{i+1}, v_{i+2}, \ldots, v_{((m+1) / 2)}, w_{i}, w_{i+1}, w_{i+2}, \ldots$, $\left.w_{((m+1) / 2)}\right\}$ and $\operatorname{LR}\left(u_{i} v_{i+1}\right)=\left\{u_{i+1}, u_{i+2}, u_{i+3}, \ldots\right.$, $u_{((m+2 i+1) / 2)}, v_{i}, v_{((m+2 i+1) / 2)}, \quad v_{((m+2 i+3) / 2)}, v_{((m+2 i+5) / 2)}$, $\ldots, v_{m}, w_{i}, \quad w_{((m+2 i+1) / 2)}, w_{((m+2 i+3) / 2)}, w_{((m+2 i+5) / 2)}$, $\left.\ldots, w_{m}\right\}$, therefore $\left|\operatorname{LR}\left(e_{i}\right)\right|=((3 m+3) / 2)$. Also, $\operatorname{LR}\left(v_{i} w_{i}\right)=V\left(R_{m}\right)$, which implies that $\mid \operatorname{LR}\left(v_{i}\right.$ $\left.w_{i}\right) \mid=3 m$. Now, $\left|\cup_{i=1}^{m} \operatorname{LR}\left(e_{i}\right)\right|=\left|V\left(R_{m}\right)\right|=3 m$.

(b) The LRN sets other than $\operatorname{LR}\left(e_{i}\right)$ and $\operatorname{LR}\left(v_{i} w_{i}\right)$ are $\operatorname{LR}\left(w_{i} w_{i+1}\right)=V\left(R_{m}\right)-\left\{u_{i+1}, v_{((m+2 i+1) / 2)}, w_{((m+2 i+1) /}\right.$ 2)\}, $\quad \operatorname{LR}\left(v_{i} v_{i+1}\right)=V\left(R_{m}\right)-\left\{u_{i+2}, v_{((m+2 i+1) / 2)}\right.$, $\left.w_{((m+2 i+1) / 2)}\right\}$, and $\operatorname{LR}\left(u_{i} u_{i+1}\right)=V\left(R_{m}\right)-$ $\left\{u_{((m+2 i+1) / 2)}, v_{i}, w_{i}\right\}$.

Now, we arrange the cardinalities of these LRN sets in Table 1.

From Table 1 , it is clear that $\left|\operatorname{LR}\left(e_{i}\right)\right| \leq|\operatorname{LR}(x)|$ $\leq\left|\operatorname{LR}\left(v_{i} w_{i}\right)\right|, \forall x \in E(\mathbb{G})$ with $1 \leq i \leq m$.

(c) Since $\left|\cup_{i=1}^{m} \operatorname{LR}\left(e_{i}\right)\right|=\left|V\left(R_{m}\right)\right|$, therefore $\mid \operatorname{LR}(x) \cap$ $\operatorname{LR}\left(e_{i}\right)|=| \operatorname{LR}(x)|\geq| \operatorname{LR}\left(e_{i}\right) \mid$.

Lemma 2. Let $R_{m}$ with $m \geq 6$ be a network of convex polytope, where $m \cong 0(\bmod 2)$. Then,

(a) $\left|L R\left(e_{i}\right)\right|=\left|\operatorname{LR}\left(v_{i} u_{i+1}\right)\right|=\left|\operatorname{LR}\left(u_{i} v_{i}\right)\right|=(3 m / 2) \quad$ and $\left|L R\left(v_{i} w_{i}\right)\right|=3 m$ with $\left|\cup_{i=1}^{m} L R\left(e_{i}\right)\right|=\left|V\left(R_{m}\right)\right|$

(b) $\left|L R\left(e_{i}\right)\right| \leq|L R(x)| \leq\left|L R\left(v_{i} w_{i}\right)\right|, \forall x \in E\left(R_{m}\right)$

(c) $\left|L R(x) \cap \cup_{i=1}^{m} L R\left(e_{i}\right)\right| \geq\left|L R\left(e_{i}\right)\right|, \forall x \in E\left(R_{m}\right)$ 
TABLE 1: Cardinalities of the LRN sets of $R_{m}$ for $m \cong 1(\bmod 2)$.

\begin{tabular}{lc}
\hline Cardinalities of LRN sets & Comparison \\
\hline$\left|\operatorname{LR}\left(w_{i} w_{i+1}\right)\right|=3 m+3$ & $3 m>3 m-3>((3 m+3) / 2)$ \\
$\left|\operatorname{LR}\left(v_{i} v_{i+1}\right)\right|=3 m+3$ & $3 m>3 m-3>((3 m+3) / 2)$ \\
$\left|\operatorname{LR}\left(u_{i} u_{i+1}\right)\right|=3 m+3$ & $3 m>3 m-3>((3 m+3) / 2)$ \\
\hline
\end{tabular}

Proof. Assume that $u_{i}, v_{i}$, and $w_{i}$ are inner, middle, and outer vertices, respectively, of $R_{m}$, where $1 \leq i \leq m$ and $m+1 \cong 1(\bmod m)$.

(a) Consider $\quad \operatorname{LR}\left(u_{i} v_{i+1}\right)=\left\{u_{i+1}, u_{i+2}, u_{i+3}, \ldots\right.$, $u_{((m+2 i) / 2)}, v_{i}, v_{((m+2 i+2) / 2)}, v_{((m+2 i+4) / 2)}, \quad v_{((m+2 i+6) / 2)}$, $\ldots, v_{m}, w_{i}, w_{((m+2 i) / 2)}, w_{((m+2 i+2) / 2)}, w_{((m+2 i+4) / 2)}, \ldots$, $\left.w_{m}\right\}, \quad \operatorname{LR}\left(u_{i} v_{i}\right)=\left\{u_{i}, u_{((m+2 i+2) / 2)}, u_{((m+2 i+4) / 2)}\right.$, $u_{((m+2 i+6) / 2)}, \ldots, u_{m}, v_{i}, v_{i+1}, v_{i+2}, \ldots, v_{(m / 2)}, v_{m}, w_{i}$, $\left.w_{i+1}, w_{i+2}, \ldots, w_{(m / 2)}\right\}$ therefore $\left|\operatorname{LR}\left(e_{i}\right)\right|=(3 m / 2)$, and $\left|\cup_{i=1}^{m} \operatorname{LR}\left(e_{i}\right)\right|=3 m$. Also, $\operatorname{LR}\left(v_{i} w_{i}\right)=V\left(R_{m}\right)$ which implies that $\left|\operatorname{LR}\left(v_{i} w_{i}\right)\right|=\left|V\left(R_{m}\right)\right|=3 m$. Furthermore, $\left|\cup_{i=1}^{m} \operatorname{LR}\left(e_{i}\right)\right|=\left|V\left(R_{m}\right)\right|=3 m$.

(b) Other LRN sets are $\operatorname{LR}\left(w_{i} w_{i+1}\right)=V\left(R_{m}\right)-$ $\left\{u_{i+1}, u_{((m+2 i+2) / 2)}\right\}, \quad \operatorname{LR}\left(v_{i} v_{(i+1)}\right)=V\left(R_{m}\right)-\left\{u_{i+1}\right.$, $\left.u_{((m+2 i+2) / 2)}\right\}$, and $\operatorname{LR}\left(u_{i} u_{(i+1)}\right)=V\left(R_{m}\right)-$ $\left\{v_{((m+2 i) / 2)}, v_{i}, w_{i}, w_{((m+2 i) / 2)}\right\}$.

The cardinalities of these LRN sets are obtained as given in Table 2.

From Table 2, it is clear that $\left|\operatorname{LR}\left(e_{i}\right)\right| \leq|\operatorname{LR}(x)|$ $\leq\left|\operatorname{LR}\left(v_{i} w_{i}\right)\right|, \forall x \in E\left(R_{m}\right)$.

(c) Since $\left|\cup_{i=1}^{m} \operatorname{LR}\left(e_{i}\right)\right|=\left|V\left(R_{m}\right)\right|$, therefore $\mid \operatorname{LR}(x) \cap$ $\cup_{i=1}^{m} \operatorname{LR}\left(e_{i}\right)|=| \operatorname{LR}(x)|\geq| \operatorname{LR}\left(e_{i}\right) \mid$.

Theorem 3. Let $R_{m}$ with $m \geq 5$ be a network of convex polytope, where $m \cong 1(\bmod 2)$. Then,

$$
1<\operatorname{dim}_{f l}\left(R_{m}\right) \leq \frac{2 m}{m+1} .
$$

Proof. To prove the result, we have following cases:

Case 1. For $m=5$, the LRN sets are as follows:

$$
\begin{aligned}
& \mathrm{LR}_{1}=\mathrm{LR}\left(u_{1} u_{2}\right)=V\left(R_{5}\right)-\left\{u_{4}, v_{1}, w_{1}\right\}, \\
& \mathrm{LR}_{2}=\mathrm{LR}\left(u_{2} u_{3}\right)=V\left(R_{5}\right)-\left\{u_{5}, v_{2}, w_{2}\right\}, \\
& \mathrm{LR}_{3}=\mathrm{LR}\left(u_{3} u_{4}\right)=V\left(R_{5}\right)-\left\{u_{1}, v_{3}, w_{3}\right\}, \\
& \mathrm{LR}_{4}=\mathrm{LR}\left(u_{4} u_{5}\right)=V\left(R_{5}\right)-\left\{u_{2}, v_{4}, w_{4}\right\}, \\
& \mathrm{LR}_{5}=\operatorname{LR}\left(u_{5} u_{1}\right)=V\left(R_{5}\right)-\left\{u_{3}, v_{5}, w_{5}\right\}, \\
& \mathrm{LR}_{6}=\operatorname{LR}\left(w_{1} w_{2}\right)=V\left(R_{5}\right)-\left\{u_{2}, v_{2}, w_{4}\right\}, \\
& \mathrm{LR}_{7}=\operatorname{LR}\left(w_{2} w_{3}\right)=V\left(R_{5}\right)-\left\{u_{3}, v_{3}, w_{5}\right\}, \\
& \mathrm{LR}_{8}=\operatorname{LR}\left(w_{3} w_{4}\right)=V\left(R_{5}\right)-\left\{u_{4}, v_{4}, w_{1}\right\}, \\
& \mathrm{LR}_{9}=\operatorname{LR}\left(w_{4} w_{4}\right)=V\left(R_{5}\right)-\left\{u_{5}, v_{5}, w_{2}\right\}, \\
& \mathrm{LR}_{10}=\operatorname{LR}\left(w_{4} w_{5}\right)=V\left(R_{5}\right)-\left\{u_{1}, v_{1}, w_{3}\right\}, \\
& \mathrm{LR}_{11}=\operatorname{LR}\left(v_{1} v_{2}\right)=V\left(R_{5}\right)-\left\{v_{4}, w_{4}\right\}, \\
& \operatorname{LR}_{12}=\operatorname{LR}\left(v_{2} v_{3}\right)=V\left(R_{5}\right)-\left\{v_{5}, w_{5}\right\},
\end{aligned}
$$

TABLE 2: Cardinalities of LRN sets of $R_{m}$ with $m \cong 0(\bmod 2)$.

\begin{tabular}{lc}
\hline Cardinalities of LRN sets & Comparison \\
\hline$\left|\operatorname{LR}\left(w_{i} w_{i+1}\right)\right|=(3 m / 2)$ & $3 m>3 m-2>(3 m / 2)$ \\
$\left|\operatorname{LR}\left(v_{i} v_{i+1}\right)\right|=(3 m / 2)$ & $3 m>3 m-2>(3 m / 2)$ \\
$\operatorname{LR}\left(u_{i} u_{i+1}\right)=(3 m / 2)$ & $3 m>3 m-4>(3 m / 2)$ \\
\hline
\end{tabular}

$$
\begin{aligned}
& \mathrm{LR}_{13}=\mathrm{LR}\left(v_{3} v_{4}\right)=V\left(R_{5}\right)-\left\{v_{1}, w_{1}\right\}, \\
& \mathrm{LR}_{14}=\mathrm{LR}\left(v_{4} v_{5}\right)=V\left(R_{5}\right)-\left\{v_{2}, w_{2}\right\}, \\
& \mathrm{LR}_{15}=\mathrm{LR}\left(v_{5} v_{1}\right)=V\left(R_{5}\right)-\left\{v_{3}, w_{1}\right\}, \\
& \mathrm{LR}_{16}=\mathrm{LR}\left(v_{1} w_{1}\right)=V\left(R_{5}\right), \\
& \mathrm{LR}_{17}=\mathrm{LR}\left(v_{2} w_{2}\right)=V\left(R_{5}\right), \\
& \mathrm{LR}_{18}=\mathrm{LR}\left(v_{3} w_{3}\right)=V\left(R_{5}\right), \\
& \mathrm{LR}_{19}=\mathrm{LR}\left(v_{4} w_{4}\right)=V\left(R_{5}\right), \\
& \mathrm{LR}_{20}=\mathrm{LR}\left(v_{5} w_{5}\right)=V\left(R_{5}\right), \\
& \mathrm{LR}_{21}=\mathrm{LR}\left(v_{1} u_{2}\right)=V\left(R_{5}\right)-\left\{v_{3}, v_{2}, u_{1}, u_{5}, w_{2}, w_{3}\right\}, \\
& \mathrm{LR}_{22}=\mathrm{LR}\left(v_{2} u_{3}\right)=V\left(R_{5}\right)-\left\{v_{3}, v_{4}, u_{1}, u_{5}, w_{2}, w_{3}\right\}, \\
& \mathrm{LR}_{23}=\mathrm{LR}\left(v_{3} u_{4}\right)=V\left(R_{5}\right)-\left\{v_{5}, v_{4}, u_{1}, u_{5}, w_{2}, w_{3}\right\}, \\
& \operatorname{LR}_{24}=\mathrm{LR}\left(v_{4} u_{5}\right)=V\left(R_{5}\right)-\left\{v_{1}, v_{5}, u_{1}, u_{5}, w_{2}, w_{3}\right\}, \\
& \operatorname{LR}_{25}=\operatorname{LR}\left(v_{5} u_{1}\right)=V\left(R_{5}\right)-\left\{v_{1}, v_{2}, u_{1}, u_{5}, w_{2}, w_{3}\right\}, \\
& \operatorname{LR}_{26}=\operatorname{LR}\left(u_{1} v_{1}\right)=V\left(R_{5}\right)-\left\{u_{2}, u_{3}, v_{2}, v_{3}, w_{4}, w_{5}\right\}, \\
& \operatorname{LR}_{27}=\operatorname{LR}\left(u_{2} v_{2}\right)=V\left(R_{5}\right)-\left\{u_{3}, u_{4}, v_{5}, v_{1}, w_{5}, w_{1}\right\}, \\
& \operatorname{LR}_{28}=\operatorname{LR}\left(u_{3} v_{3}\right)=V\left(R_{5}\right)-\left\{u_{4}, u_{5}, v_{1}, v_{2}, w_{3}, w_{2}\right\}, \\
& \operatorname{LR}_{29}=\operatorname{LR}\left(u_{4} v_{4}\right)=V\left(R_{5}\right)-\left\{u_{5}, u_{1}, v_{2}, v_{3}, w_{2}, w_{1}\right\}, \\
& \operatorname{LR}_{30}=\operatorname{LR}\left(u_{5} v_{5}\right)=V\left(R_{5}\right)-\left\{u_{1}, u_{2}, v_{3}, v_{4}, w_{3}, w_{2}\right\} .
\end{aligned}
$$

As, for $1 \leq i \leq 5,\left|\operatorname{LR}\left(e_{i}\right)\right|=\left|\operatorname{LR}\left(u_{i} u_{i}\right)\right|=\left|\operatorname{LR}\left(v_{i} u_{i+1}\right)\right|$ $=9, \quad\left|\operatorname{LR}\left(e_{i}\right)\right| \leq|\operatorname{LR}(x)|, \quad \forall x \in E\left(R_{5}\right)$. Furthermore, $\left|\cup_{i=1}^{5} \operatorname{LR}\left(v_{i} u_{i+1}\right)\right|=15$ and $\left|\operatorname{LR}(x) \cap \cup_{i=1}^{5} \operatorname{LR}\left(e_{i}\right)\right|=9$, $\forall x \in E\left(R_{5}\right)$. There exists an upper LRF $\phi: V$ $\left(R_{5}\right) \longrightarrow[0,1]$ defined as $\phi(v)=(1 / 9), \forall v \in V\left(R_{5}\right)$. Consequently, by Theorem $1, \operatorname{dim}_{f l}\left(R_{5}\right) \leq(5 / 3)$. Similarly, for $1 \leq i \leq 5,\left|\operatorname{LR}\left(v_{i} w_{i}\right)\right|=15$ and $\mid \operatorname{LR}\left(v_{i}\right.$ $\left.w_{i}\right)|\geq| \operatorname{LR}(x) \mid, \forall x \in E\left(R_{5}\right)$. Thus, there exists a lower LRF such that $\phi^{\prime}: V\left(R_{5}\right) \longrightarrow[0,1]$ defined as $\phi^{\prime}(v)=(1 / 15), \forall v \in V\left(R_{5}\right)$. Consequently, by Theorem $2, \operatorname{dim}_{f l}\left(R_{5}\right) \geq 1$. Since $R_{5}$ is a nonbipartite network, therefore $\operatorname{dim}_{f l}\left(R_{5}\right)$ must be greater than 1 . Hence, we have

$$
1<\operatorname{dim}_{f l}\left(R_{5}\right) \leq \frac{5}{3}
$$

Case 2. For $m>5$, by Lemma 1, $\left|\operatorname{LR}\left(e_{i}\right)\right|=((3 m+3) / 2)$ and $\left|\operatorname{LR}\left(e_{i}\right)\right| \leq|\operatorname{LR}(x)|, \quad \forall x \in E\left(R_{m}\right)$. Similarly, $\left|\operatorname{LR}\left(u_{i} w_{i}\right)\right|=3 m$ and $\left|\operatorname{LR}\left(u_{i} w_{i}\right)\right| \geq|\operatorname{LR}(x)|, \quad \forall x \in E$ $\left(R_{m}\right)$. Therefore, $\phi: V\left(R_{m}\right) \longrightarrow[0,1]$ is defined by $\phi(v)=(2 /(3 m+3)), \forall v \in V\left(R_{m}\right)$ is an upper LRF and $\phi^{\prime}: V\left(R_{m}\right) \longrightarrow[0,1] \quad$ is defined by $\phi^{\prime}(v)=$ $(1 / 3 m), \forall v \in V\left(R_{m}\right)$ is a lower LRF. Moreover, $R_{m}$ is a nonbipartite network. Therefore, by Theorems 1 and 2, we have 


$$
1<\operatorname{dim}_{f l}\left(R_{m}\right) \leq \frac{2 m}{m+1} .
$$

Theorem 4. Let $R_{m}$ with $m \geq 6$ be a network of convex polytope, where $m \cong 0(\bmod 2)$. Then,

$$
1<\operatorname{dim}_{f l}\left(R_{m}\right) \leq 2 .
$$

Proof. To prove the result, we have following two cases:

Case 1. For $m=6$, the LRN sets are as follows:

$$
\begin{aligned}
& \mathrm{LR}_{1}=\operatorname{LR}\left(u_{1} u_{2}\right)=V\left(R_{6}\right)-\left\{v_{1}, v_{4}, w_{1}, w_{4}\right\}, \\
& \operatorname{LR}_{2}=\operatorname{LR}\left(u_{2} u_{3}\right)=V\left(R_{6}\right)-\left\{v_{5}, v_{2}, w_{2}, w_{5}\right\} \text {, } \\
& \mathrm{LR}_{3}=\operatorname{LR}\left(u_{3} u_{4}\right)=V\left(R_{6}\right)-\left\{v_{3}, v_{6}, w_{3}, w_{6}\right\}, \\
& \mathrm{LR}_{4}=\operatorname{LR}\left(u_{4} u_{5}\right)=V\left(R_{6}\right)-\left\{v_{4}, v_{1}, w_{4}, w_{1}\right\}, \\
& \operatorname{LR}_{5}=\operatorname{LR}\left(u_{5} u_{6}\right)=V\left(R_{6}\right)-\left\{v_{5}, v_{2}, w_{5}, w_{2}\right\} \text {, } \\
& \operatorname{LR}_{6}=\operatorname{LR}\left(u_{6} u_{1}\right)=V\left(R_{6}\right)-\left\{v_{6}, v_{3}, w_{6}, w_{3}\right\} \text {, } \\
& \operatorname{LR}_{7}=\operatorname{LR}\left(w_{1} w_{2}\right)=V\left(R_{6}\right)-\left\{u_{2}, u_{5}, w_{2}, w_{5}\right\}, \\
& \operatorname{LR}_{8}=\operatorname{LR}\left(w_{2} w_{3}\right)=V\left(R_{6}\right)-\left\{u_{3}, u_{6}, w_{3}, w_{6}\right\} \text {, } \\
& \operatorname{LR}_{9}=\operatorname{LR}\left(w_{3} w_{4}\right)=V\left(R_{6}\right)-\left\{u_{4}, u_{1}, w_{4}, w_{1}\right\}, \\
& \operatorname{LR}_{10}=\operatorname{LR}\left(w_{4} w_{5}\right)=V\left(R_{6}\right)-\left\{u_{5}, u_{2}, w_{5}, w_{2}\right\} \text {, } \\
& \operatorname{LR}_{11}=\operatorname{LR}\left(w_{5} w_{6}\right)=V\left(R_{6}\right)-\left\{u_{6}, u_{3}, w_{6}, w_{3}\right\} \text {, } \\
& \operatorname{LR}_{12}=\operatorname{LR}\left(w_{6} w_{1}\right)=V\left(R_{6}\right)-\left\{u_{1}, u_{4}, w_{1}, w_{4}\right\} \text {, } \\
& \mathrm{LR}_{13}=\operatorname{LR}\left(v_{1} v_{2}\right)=V\left(R_{6}\right)-\left\{u_{2}, u_{5}\right\} \text {, } \\
& \operatorname{LR}_{14}=\operatorname{LR}\left(v_{2} v_{3}\right)=V\left(R_{6}\right)-\left\{u_{3}, u_{6}\right\} \text {, } \\
& \operatorname{LR}_{15}=\operatorname{LR}\left(v_{3} v_{4}\right)=V\left(R_{6}\right)-\left\{u_{4}, u_{1}\right\} \text {, } \\
& \operatorname{LR}_{16}=\operatorname{LR}\left(v_{4} v_{5}\right)=V\left(R_{6}\right)-\left\{u_{5}, u_{2}\right\}, \\
& \operatorname{LR}_{17}=\operatorname{LR}\left(v_{5} v_{6}\right)=V\left(R_{6}\right)-\left\{u_{6}, u_{3}\right\} \text {, } \\
& \mathrm{LR}_{18}=\operatorname{LR}\left(v_{6} v_{1}\right)=V\left(R_{6}\right)-\left\{u_{1}, u_{4}\right\}, \\
& \mathrm{LR}_{19}=\operatorname{LR}\left(v_{1} w_{1}\right)=V\left(R_{6}\right), \\
& \mathrm{LR}_{20}=\operatorname{LR}\left(v_{2} w_{2}\right)=V\left(R_{6}\right) \text {, } \\
& \mathrm{LR}_{21}=\operatorname{LR}\left(v_{3} w_{3}\right)=V\left(R_{6}\right), \\
& \mathrm{LR}_{22}=\operatorname{LR}\left(v_{4} w_{4}\right)=V\left(R_{6}\right), \\
& \mathrm{LR}_{23}=\operatorname{LR}\left(v_{5} w_{5}\right)=V\left(R_{6}\right) \text {, } \\
& \mathrm{LR}_{24}=\operatorname{LR}\left(v_{6} w_{6}\right)=V\left(R_{6}\right) \text {, } \\
& \operatorname{LR}_{25}=\operatorname{LR}\left(u_{1} v_{1}\right)=\left\{u_{1}, u_{5}, u_{6}, v_{1}, v_{2}, v_{3}, w_{1}, w_{2}, w_{3}\right\} \text {, } \\
& \operatorname{LR}_{26}=\operatorname{LR}\left(u_{2} v_{2}\right)=\left\{u_{2}, u_{6}, u_{1}, v_{2}, v_{3}, v_{4}, w_{2}, w_{3}, w_{4}\right\} \text {, } \\
& \operatorname{LR}_{27}=\operatorname{LR}\left(u_{3} v_{3}\right)=\left\{u_{3}, u_{1}, u_{2}, v_{3}, v_{4}, v_{5}, w_{3}, w_{4}, w_{5}\right\} \text {, } \\
& \operatorname{LR}_{28}=\operatorname{LR}\left(u_{4} v_{4}\right)=\left\{u_{4}, u_{2}, u_{3}, v_{4}, v_{5}, v_{6}, w_{4}, w_{5}, w_{6}\right\} \text {, } \\
& \operatorname{LR}_{29}=\operatorname{LR}\left(u_{5} v_{5}\right)=\left\{u_{5}, u_{3}, u_{4}, v_{5}, v_{6}, v_{1}, w_{5}, w_{6}, w_{1}\right\} \text {, } \\
& \operatorname{LR}_{30}=\operatorname{LR}\left(u_{6} v_{6}\right)=\left\{u_{6}, u_{4}, u_{5}, v_{6}, v_{1}, v_{2}, w_{6}, w_{1}, w_{2}\right\} \text {, } \\
& \operatorname{LR}_{31}=\operatorname{LR}\left(v_{1} u_{2}\right)=\left\{u_{2}, u_{3}, u_{4}, v_{1}, v_{5}, v_{6}, w_{1}, w_{5}, w_{6}\right\} \text {, }
\end{aligned}
$$

$$
\begin{aligned}
& \operatorname{LR}_{32}=\operatorname{LR}\left(v_{2} u_{3}\right)=\left\{u_{3}, u_{4}, u_{5}, v_{1}, v_{2}, v_{6}, w_{1}, w_{2}, w_{6}\right\}, \\
& \operatorname{LR}_{33}=\operatorname{LR}\left(v_{3} u_{4}\right)=\left\{u_{4}, u_{5}, u_{6}, v_{1}, v_{2}, v_{3}, w_{1}, w_{3}, w_{5}\right\}, \\
& \operatorname{LR}_{34}=\operatorname{LR}\left(v_{4} u_{5}\right)=\left\{u_{5}, u_{6}, u_{1}, v_{2}, v_{3}, v_{4}, w_{4}, w_{2}, w_{3}\right\}, \\
& \operatorname{LR}_{35}=\operatorname{LR}\left(v_{5} u_{6}\right)=\left\{u_{6}, u_{1}, u_{2}, v_{3}, v_{4}, v_{5}, w_{3}, w_{4}, w_{5}\right\}, \\
& \operatorname{LR}_{36}=\operatorname{LR}\left(v_{6} u_{1}\right)=\left\{u_{1}, u_{2}, u_{3}, v_{4}, v_{5}, v_{6}, w_{4}, w_{5}, w_{6}\right\} .
\end{aligned}
$$

For $1 \leq i \leq 6, \quad\left|\operatorname{LR}\left(e_{i}\right)\right|=\left|\operatorname{LR}\left(u_{i} u_{i}\right)\right|=\left|\operatorname{LR}\left(v_{i} u_{i+1}\right)\right|=9$ and $\left|\operatorname{LR}\left(e_{i}\right)\right| \leq|\operatorname{LR}(x)|, \quad \forall x \in E\left(R_{6}\right)$. Furthermore, $\left|\cup{ }_{i=1}^{6} \operatorname{LR}\left(e_{i}\right)\right|=18$ and $\left|\operatorname{LR}(x) \cap \cup_{i=1}^{6} \operatorname{LR}\left(e_{i}\right)\right|=9$, $\forall x \in E\left(R_{6}\right)$. Therefore, there exists a lower LRF $\phi: V\left(R_{6}\right) \longrightarrow[0,1]$ defined as $\phi(v)=(1 / 9)$ for each $v \in V\left(R_{m}\right)$. Consequently, by Theorem 1, $\operatorname{dim}_{f l}\left(R_{6}\right) \leq 2$.

Similarly, for $1 \leq i \leq 6, \quad\left|\operatorname{LR}\left(v_{i} w_{i}\right)\right|=18 \quad$ and $\left|\operatorname{LR}\left(v_{i} w_{i}\right)\right| \geq|\operatorname{LR}(x)|, \forall x \in E\left(R_{6}\right)$. Thus, there exists an upper LRF $\phi^{\prime}: V\left(R_{6}\right) \longrightarrow[0,1]$ defined as $\phi(v)=(1 / 18)$ for each $v \in V\left(R_{m}\right)$. Consequently, by Theorem 2, $\operatorname{dim}_{f l}\left(R_{6}\right) \geq 1$. Furthermore, $R_{6}$ is a nonbipartite network, so $\operatorname{dim}_{f l}\left(R_{6}\right)$ must be greater than 1 . Hence, we have

$$
1<\operatorname{dim}_{f l}\left(R_{6}\right) \leq 2 .
$$

Case 2. For $m>6$, by Lemma 2, $\left|\operatorname{LR}\left(e_{i}\right)\right|=(3 m / 2)$ for $1 \leq i \leq m$ and $\left|\operatorname{LR}\left(e_{i}\right)\right| \leq|\operatorname{LR}(x)|, \forall x \in E\left(R_{m}\right)$. Therefore, $\phi: V\left(R_{m}\right) \longrightarrow[0,1]$ defined by $\phi(v)=(2 / 3 m)$, $\forall v \in V\left(R_{m}\right)$ is an upper LRF. Thus, by Theorem 1 , the $\operatorname{dim}_{f l}\left(R_{m}\right) \leq 2$. Similarly, as $\left|\operatorname{LR}\left(v_{i} w_{i}\right)\right|=3 m$ and $\left|\operatorname{LR}\left(v_{i} w_{i}\right)\right| \geq|\operatorname{LR}(x)|, \quad \forall x \in E\left(R_{m}\right), \quad$ where $1 \leq i \leq m$. Therefore, $\phi^{\prime}: V\left(R_{m}\right) \longrightarrow[0,1]$ defined by $\phi^{\prime}(v)=(1 / 3 m), \forall v \in V\left(R_{m}\right)$ is a lower LRF. Thus, by Theorem 2, $\operatorname{dim}_{f l}\left(R_{m}\right) \geq 1$. Moreover, as $R_{m}$ is a nonbipartite network, it implies that $\operatorname{dim}_{f l}\left(R_{m}\right)$ must be greater than 1 . Hence, we have

$$
1<\operatorname{dim}_{f l}\left(R_{m}\right) \leq 2 .
$$

3.2. LRN Sets and LFMD of Convex Polytope $A_{m}$. This subsection deals with the results for the LRN sets and LFMD of the convex polytopes network $A_{m}$ with $m \geq 5$.

Lemma 3. Let $A_{m}$ be a network of convex polytope, where $m \geq 5$ and $m \cong 1(\bmod 2)$. Then, for $1 \leq i \leq m$,

(a) $\left|\operatorname{LR}\left(e_{i}\right)\right|=\left|\operatorname{LR}\left(v_{i} u_{i}\right)\right|=\left|\operatorname{LR}\left(v_{i} u_{i+1}\right)\right|=\left|\operatorname{LR}\left(v_{i} w_{i+1}\right)\right|=$ $=\left|L R\left(v_{i} w_{i}\right)\right|=((3 m+3) / 2)$ and $\left|L R\left(v_{i} v_{i+1}\right)\right|=3 m-3$ with $\left|\cup_{i=1}^{m} \operatorname{LR}\left(e_{i}\right)\right|=\left|V\left(R_{m}\right)\right|=3 m$

(b) $\left|L R\left(e_{i}\right)\right| \leq|\operatorname{LR}(x)| \leq\left|\operatorname{LR}\left(v_{i} v_{i+1}\right)\right|, \forall x \in E\left(A_{m}\right)$

(c) $\left|L R(x) \cap \cup_{i=1}^{m} L R\left(e_{i}\right)\right| \geq\left|L R\left(e_{i}\right)\right|, \forall x \in E\left(A_{m}\right)$

Proof. Assume that $u_{i}, v_{i}$, and $w_{i}$ are inner, middle, and outer vertices of $A_{m}$, respectively, where $1 \leq i \leq m$ and $m+1 \cong 1(\bmod m)$. 
(a) Since $\operatorname{LR}\left(v_{i} u_{i+1}\right)=\left\{u_{i+1}, u_{i+2}, u_{i+3}, \ldots, u_{((m+2 i+1) / 2)}\right.$, $v_{i}, \quad v_{((m+2 i+1) / 2)}, v_{((m+2 i+3) / 2)}, \ldots, v_{m}, w_{i}, w_{i+1}, w_{i+2}$, $\left.\ldots, w_{((m+2 i+1) / 2)}, w_{((m+2 i+5) / 2)}, \ldots, w_{m}\right\}, \operatorname{LR}\left(v_{i} w_{i}\right)=$ $\left\{u_{i}, u_{i+1}, u_{i+2}, \quad u_{i+3}, \ldots, u_{((m+2 i-1) / 2)}, v_{i}, v_{i+1}, v_{i+2}, \ldots\right.$, $\left.v_{((m+2 i-1) / 2)}, w_{i}, \quad w_{((m+2 i+1) / 2)}, w_{((m+2 i+3) / 2)}, \ldots, w_{m}\right\}$, $\operatorname{LR}\left(u_{i} v_{i}\right)=\left\{u_{i}, u_{((m+2 i+1) / 2)}, \quad u_{((m+2 i+3) / 2)}, \ldots, u_{m}\right.$, $v_{i}, v_{i+1}, \quad v_{i+2}, \ldots, v_{((m+2 i-1) / 2)}, w_{i}, w_{i+1}, w_{i+2}, \ldots \ldots$, $\left.w_{((m+2 i-1) / 2)}\right\}$, and $\operatorname{LR}\left(v_{i} w_{i+1}\right)=\left\{u_{i}, u_{i+1}, u_{((m+6 i-2) / 2)}\right.$, $u_{((m+6 i-2) / 2)+1}, \ldots, u_{m}, v_{i}, \quad v_{((m+6 i-2) / 2)}, \quad v_{((m+6 i-2) / 2)+1}$, $\left.\ldots, v_{m} w_{i+1}, w_{i+2}, w_{i+3}, \ldots, w_{((m+6 i-4) / 2)}\right\}$, therefore $\left|\operatorname{LR}\left(e_{i}\right)\right|=\left|\operatorname{LR}\left(v_{i} u_{i}\right)\right|=\left|\operatorname{LR}\left(v_{i} u_{i+1}\right)\right|=\left|\operatorname{LR}\left(v_{i} w_{i+1}\right)\right|=$ $\left|\operatorname{LR}\left(v_{i} w_{i}\right)\right|=((3 m+3) / 2) \quad$ with $\left|\cup_{i=1}^{m} \operatorname{LR}\left(e_{i}\right)\right|=$ $\left|V\left(R_{m}\right)\right|=3 m$. Also, =LR $\left(v_{i} v_{i+1}\right)=V\left(A_{m}\right)-\left\{u_{i}\right.$, $\left.v_{((m+2 i+1) / 2)}, w_{i}\right\}$; therefore, $\left|\operatorname{LR}\left(v_{i} v_{i+1}\right)\right|=\left|V\left(R_{m}\right)\right|-$ $3=3 m-3$.

(b) The LRN sets of the other $\operatorname{LR}\left(e_{i}\right)$ and $\operatorname{LR}\left(v_{i} v_{i+1}\right)$ are $\operatorname{LR}\left(w_{i} w_{i+1}\right)=V\left(A_{m}\right)-\left\{u_{i}, u_{i+1}, u_{((m+2 i-1) / 2)}\right.$, $\left.v_{i}, w_{((m+2 i-1) / 2)}\right\}$ and $\operatorname{LR}\left(u_{i} u_{i+1}\right)=V\left(A_{m}\right)-\left\{u_{i}, u_{i+1}\right.$, $\left.u_{((m+2 i+1) / 2)}, v_{i}, w_{((m+2 i+1) / 2)}\right\}$.

Now, we arrange the cardinalities of these LRN set in Table 3.

From Table 3, it is clear that $\left|\mathrm{LR}\left(e_{i}\right)\right| \leq|\operatorname{LR}(x)| \leq$ $\left|\operatorname{LR}\left(v_{i} v_{i+1}\right)\right|, \forall x \in E\left(A_{m}\right)$.

(c) Since $\left|\cup_{i=1}^{m} \operatorname{LR}\left(e_{i}\right)\right|=\left|V\left(A_{m}\right)\right|$, therefore $\mid \operatorname{LR}(x) \cap$ $\cup_{i=1}^{m} \operatorname{LR}\left(e_{i}\right)|=| \operatorname{LR}(x)|\geq| \operatorname{LR}\left(e_{i}\right) \mid$.

Lemma 4. Let $A_{m}$ be a network of convex polytope, where $m \geq 6$ and $m \cong 0(\bmod 2)$. Then, for $1 \leq i \leq m$,

(a) $\left|\operatorname{LR}\left(e_{i}\right)\right|=\left|\operatorname{LR}\left(v_{i} u_{i}\right)\right|=\left|\operatorname{LR}\left(v_{i} u_{i+1}\right)\right|=\left|\operatorname{LR}\left(v_{i} w_{i+1}\right)\right|=$ $\left|L R\left(v_{i} w_{i}\right)\right|=((3 m+2) / 2)$ and $\left|L R\left(v_{i} v_{i+1}\right)\right|=3 m-3$ with $\left|\cup_{i=1}^{m} L R\left(e_{i}\right)\right|=3 m$

(b) $\left|\operatorname{LR}\left(e_{i}\right)\right| \leq|\operatorname{LR}(x)| \leq\left|\operatorname{LR}\left(v_{i} v_{i+1}\right)\right|, \forall x \in E\left(A_{m}\right)$

(c) $\left|L R(x) \cap \cup_{i=1}^{m} L R\left(e_{i}\right)\right|=|L R(x)| \geq\left|L R\left(e_{i}\right)\right|, \forall x \in E$ $\left(A_{m}\right)$

Proof. Assume that $u_{i}, v_{i}$, and $w_{i}$ are inner, middle, and outer vertices of $A_{m}$, respectively, where $1 \leq i \leq m$ and $m+1 \cong 1(\bmod m)$.

(a) Consider $\quad \operatorname{LR}\left(v_{i} u_{i+1}\right)=\left\{u_{i+1}, u_{i+2}, u_{i+3}, \ldots\right.$, $u_{((m+2 i) / 2)}, \quad v_{i}, v_{((m+6 i-2) / 2)}, v_{((m+6 i-2) / 2)+1}, \ldots, v_{m}, w_{i}$, $\left.w_{i+1}, w_{i+2}, \ldots, w_{((m+6 i-2) / 2)}, w_{((m+6 i-2) / 2)+1}, \ldots, w_{m}\right\}$, $\operatorname{LR}\left(v_{i} w_{i}\right)=\left\{u_{i}, u_{i+1}, u_{i+2}, u_{i+3}, \ldots, u_{((m+4 i-2) / 2)}, \quad v_{i}\right.$, $v_{i+1}, v_{i+2}, \ldots, v_{((m+2 i-2) / 2)}, \quad w_{i}, w_{((m+6 i-2) / 2)}$, $\left.w_{((m+6 i) / 2)}, \ldots, w_{m}\right\}, \quad \operatorname{LR}\left(u_{i} v_{i}\right)=\left\{u_{i}, u_{((m+6 i-2) / 2)}\right.$, $u_{((m+6 i-2) / 2)+1}, \ldots, u_{m}, \quad v_{i}, v_{i+1}, v_{i+2}, \ldots, v_{((m+2 i-2) / 2)}$, $\left.w_{i}, w_{i+1}, w_{i+2}, \ldots, w_{((m+2 i-2) / 2)}\right\}, \quad \operatorname{LR}\left(v_{i} w_{i+1}\right)=\left\{u_{i}\right.$, $u_{i+1}, u_{((m+6 i-2) / 2)}, u_{((m+6 i) / 2)}, \ldots, u_{m}, v_{i}, v_{((m+6 i-2) / 2)}$, $\left.v_{((m+6 i) / 2)}, \ldots, v_{m}, w_{i+1}, w_{i+2}, w_{i+3}, \ldots, w_{((m+6 i-4) / 2)}\right\}$, and $\operatorname{LR}\left(v_{i} v_{i+1}\right)=V\left(A_{m}\right)-\left\{u_{i+1}, w_{i+2}, w_{((m+6 i-2) / 2)}\right\}$. Furthermore, $\left|\operatorname{LR}\left(e_{i}\right)\right|=((3 m+2) / 2)$ and |LR $\left(v_{i} v_{i+1}\right) \mid=3 m-3$ with $\left|\cup_{i=1}^{m} \operatorname{LR}\left(e_{i}\right)\right|=\left|V\left(A_{m}\right)\right|=$ $3 m$.

(b) The LRN sets of $A_{m}$ other than $\operatorname{LR}\left(e_{i}\right)$ and $\operatorname{LR}\left(v_{i} v_{i+1}\right)$ are $\operatorname{LR}\left(w_{i} w_{i+1}\right)=V\left(A_{m}\right)-\left\{u_{i}, u_{i+1}, v_{i}, v_{((m+2 i) / 2)}\right\}$ and $\operatorname{LR}\left(u_{i} u_{(i+1)}\right)=V\left(A_{m}\right)-\left\{v_{((m+4 i-2) / 2)}, v_{i}, w_{i}\right.$,
TABLE 3: Cardinalities of LRN sets of $A_{m}$ for $m \cong 1(\bmod 2)$.

\begin{tabular}{lc}
\hline Cardinalities of LRN sets & Comparison \\
\hline$\left|\operatorname{LR}\left(w_{i} w_{i+1}\right)\right|=3 m-5$ & $3 m>3 m-5>((3 m+3) / 2)$ \\
$\left|\operatorname{LR}\left(u_{i} u_{i+1}\right)\right|=3 m-5$ & $3 m>3 m-5>((3 m+3) / 2)$ \\
\hline
\end{tabular}

$\left.w_{i+1}\right\}$. Now, we arrange cardinalities of these LRN set in Table 4.

From Table 4 , it is clear that $\left|\operatorname{LR}\left(e_{i}\right)\right| \leq|\operatorname{LR}(x)| \leq$ $\left|\operatorname{LR}\left(v_{i} v_{i+1}\right)\right|, \forall x \in E\left(A_{m}\right)$.

(c) Since $\left|\cup_{i=1}^{m} \operatorname{LR}\left(e_{i}\right)\right|=\left|V\left(A_{m}\right)\right|$, therefore $\mid \operatorname{LR}(x) \cap$ $\cup_{i=1}^{m} \operatorname{LR}\left(e_{i}\right)|=| \operatorname{LR}(x)|\geq| \operatorname{LR}\left(e_{i}\right) \mid$.

Theorem 5. Let $A_{m}$ with $m \geq 5$ be a network of convex polytope, where $m \cong 1(\bmod 2)$. Then,

$$
\frac{m}{m-1} \leq \operatorname{dim}_{f l}\left(A_{m}\right) \leq \frac{2 m}{m+1}
$$

Proof. To prove the result, we have following cases:

Case 1. For $m=5$, the LRN sets are as follows:

$$
\begin{aligned}
& \mathrm{LR}_{1}=\operatorname{LR}\left(u_{1} v_{1}\right)=V\left(A_{5}\right)-\left\{u_{2}, u_{3}, v_{4}, v_{5}, w_{4}, w_{5}\right\}, \\
& \mathrm{LR}_{2}=\operatorname{LR}\left(u_{2} v_{2}\right)=V\left(A_{5}\right)-\left\{u_{3}, u_{4}, v_{1}, v_{2}, w_{1}, w_{2}\right\} \text {, } \\
& \mathrm{LR}_{3}=\operatorname{LR}\left(u_{3} v_{3}\right)=V\left(A_{5}\right)-\left\{u_{5}, u_{1}, v_{3}, v_{4}, w_{3}, w_{4}\right\} \text {, } \\
& \operatorname{LR}_{4}=\operatorname{LR}\left(u_{4} v_{4}\right)=V\left(A_{5}\right)-\left\{u_{2}, u_{3}, v_{5}, v_{1}, w_{5}, w_{1}\right\} \text {, } \\
& \mathrm{LR}_{5}=\operatorname{LR}\left(u_{5} v_{5}\right)=V\left(A_{5}\right)-\left\{u_{4}, u_{5}, v_{2}, v_{3}, w_{2}, w_{3}\right\} \text {, } \\
& \mathrm{LR}_{6}=\operatorname{LR}\left(v_{1} u_{2}\right)=V\left(A_{5}\right)-\left\{u_{1}, u_{2}, v_{2}, v_{3}, w_{3}, w_{4}\right\} \text {, } \\
& \operatorname{LR}_{7}=\operatorname{LR}\left(v_{2} u_{3}\right)=V\left(A_{5}\right)-\left\{u_{2}, u_{3}, v_{3}, v_{4}, w_{4}, w_{5}\right\} \text {, } \\
& \operatorname{LR}_{8}=\operatorname{LR}\left(v_{3} u_{4}\right)=V\left(A_{5}\right)-\left\{u_{3}, u_{4}, v_{4}, v_{5}, w_{5}, w_{1}\right\} \text {, } \\
& \mathrm{LR}_{9}=\operatorname{LR}\left(v_{4} u_{5}\right)=V\left(A_{5}\right)-\left\{u_{4}, u_{5}, v_{5}, v_{1}, w_{1}, w_{2}\right\} \text {, } \\
& \operatorname{LR}_{10}=\operatorname{LR}\left(v_{5} u_{1}\right)=V\left(A_{5}\right)-\left\{u_{5}, u_{1}, v_{1}, v_{2}, w_{2}, w_{3}\right\} \text {, } \\
& \operatorname{LR}_{11}=\operatorname{LR}\left(v_{1} w_{1}\right)=V\left(A_{5}\right)-\left\{u_{4}, u_{5}, v_{4}, v_{5}, w_{2}, w_{3}\right\} \text {, } \\
& \operatorname{LR}_{12}=\operatorname{LR}\left(v_{2} w_{2}\right)=V\left(A_{5}\right)-\left\{u_{5}, u_{1}, v_{5}, v_{1}, w_{3}, w_{4}\right\} \text {, } \\
& \operatorname{LR}_{13}=\operatorname{LR}\left(v_{3} w_{3}\right)=V\left(A_{5}\right)-\left\{u_{1}, u_{2}, v_{1}, v_{2}, w_{4}, w_{5}\right\} \text {, } \\
& \mathrm{LR}_{14}=\operatorname{LR}\left(v_{4} w_{4}\right)=V\left(A_{5}\right)-\left\{u_{2}, u_{3}, v_{2}, v_{3}, w_{5}, w_{1}\right\} \text {, } \\
& \operatorname{LR}_{15}=\operatorname{LR}\left(v_{5} w_{5}\right)=V\left(A_{5}\right)-\left\{u_{3}, u_{4}, v_{3}, v_{4}, w_{1}, w_{5}\right\} \text {, } \\
& \operatorname{LR}_{16}=\operatorname{LR}\left(v_{1} w_{2}\right)=V\left(A_{5}\right)-\left\{u_{3}, u_{4}, v_{2}, v_{3}, w_{1}, w_{5}\right\} \text {, } \\
& \mathrm{LR}_{17}=\operatorname{LR}\left(v_{2} w_{3}\right)=V\left(A_{5}\right)-\left\{u_{4}, u_{5}, v_{3}, v_{4}, w_{2}, w_{1}\right\} \text {, } \\
& \operatorname{LR}_{18}=\operatorname{LR}\left(v_{3} w_{4}\right)=V\left(A_{5}\right)-\left\{u_{5}, u_{1}, v_{4}, v_{5}, w_{3}, w_{2}\right\} \text {, } \\
& \operatorname{LR}_{19}=\operatorname{LR}\left(v_{4} w_{5}\right)=V\left(A_{5}\right)-\left\{u_{1}, u_{2}, v_{5}, v_{1}, w_{4}, w_{3}\right\} \text {, } \\
& \mathrm{LR}_{20}=\operatorname{LR}\left(v_{5} w_{1}\right)=V\left(A_{5}\right)-\left\{u_{2}, u_{3}, v_{1}, v_{2}, w_{5}, w_{4}\right\} \text {, } \\
& \operatorname{LR}_{21}=\operatorname{LR}\left(u_{1} u_{2}\right)=V\left(A_{5}\right)-\left\{u_{4}, v_{1}, w_{1}, w_{2}, w_{4}\right\}, \\
& \operatorname{LR}_{22}=\operatorname{LR}\left(u_{2} u_{3}\right)=V\left(A_{5}\right)-\left\{u_{5}, v_{2}, w_{2}, w_{3}, w_{5}\right\} \text {, } \\
& \mathrm{LR}_{23}=\operatorname{LR}\left(u_{3} u_{4}\right)=V\left(A_{5}\right)-\left\{u_{1}, v_{3}, w_{1}, w_{3}, w_{4}\right\}, \\
& \operatorname{LR}_{24}=\operatorname{LR}\left(u_{4} u_{5}\right)=V\left(A_{5}\right)-\left\{u_{2}, v_{4}, w_{1}, w_{4}, w_{2}\right\} \text {, }
\end{aligned}
$$


TABLE 4: Cardinalities of LRN sets of $A_{m}$ for $m \cong 0(\bmod 2)$.

\begin{tabular}{lc}
\hline Cardinalities of LRN sets & Comparison \\
\hline$\left|\operatorname{LR}\left(w_{i} w_{i+1}\right)\right|=3 m-4$ & $3 m>3 m-4>((3 m+2) / 2)$ \\
$\left|\operatorname{LR}\left(u_{i} u_{i+1}\right)\right|=3 m-4$ & $3 m>3 m-4>((3 m+2) / 2)$ \\
\hline
\end{tabular}

$$
\begin{aligned}
& \operatorname{LR}_{25}=\operatorname{LR}\left(u_{5} u_{1}\right)=V\left(A_{5}\right)-\left\{u_{3}, v_{5}, w_{5}, w_{1}, w_{3}\right\}, \\
& \operatorname{LR}_{26}=\operatorname{LR}\left(w_{1} w_{2}\right)=V\left(A_{5}\right)-\left\{u_{1}, u_{2}, u_{4}, v_{1}, w_{4}\right\}, \\
& \operatorname{LR}_{27}=\operatorname{LR}\left(w_{2} w_{3}\right)=V\left(A_{5}\right)-\left\{u_{2}, u_{3}, u_{5}, v_{2}, w_{5}\right\}, \\
& \operatorname{LR}_{28}=\operatorname{LR}\left(w_{3} w_{4}\right)=V\left(A_{5}\right)-\left\{u_{3}, u_{4}, u_{1}, v_{3}, w_{1}\right\}, \\
& \operatorname{LR}_{29}=\operatorname{LR}\left(w_{4} w_{5}\right)=V\left(A_{5}\right)-\left\{u_{4}, u_{5}, u_{2}, v_{4}, w_{2}\right\}, \\
& \operatorname{LR}_{30}=\operatorname{LR}\left(w_{5} w_{1}\right)=V\left(A_{5}\right)-\left\{u_{5}, u_{1}, u_{3}, v_{5}, w_{3}\right\}, \\
& \operatorname{LR}_{31}=\operatorname{LR}\left(v_{1} v_{2}\right)=V\left(A_{5}\right)-\left\{u_{2}, w_{2}, v_{4}\right\}, \\
& \operatorname{LR}_{32}=\operatorname{LR}\left(v_{2} v_{3}\right)=V\left(A_{5}\right)-\left\{u_{3}, w_{3}, v_{5}\right\}, \\
& \operatorname{LR}_{33}=\operatorname{LR}\left(v_{3} v_{4}\right)=V\left(A_{5}\right)-\left\{u_{4}, w_{4}, v_{1}\right\}, \\
& \operatorname{LR}_{34}=\operatorname{LR}\left(v_{4} v_{5}\right)=V\left(A_{5}\right)-\left\{u_{5}, w_{5}, v_{2}\right\}, \\
& \operatorname{LR}_{35}=\operatorname{LR}\left(v_{5} v_{1}\right)=V\left(A_{5}\right)-\left\{u_{6}, w_{6}, v_{3}\right\} .
\end{aligned}
$$

For $\quad 1 \leq i \leq 5, \quad\left|\operatorname{LR}\left(e_{i}\right)\right|=\left|\operatorname{LR}\left(v_{i} w_{i}\right)\right|=\left|\operatorname{LR}\left(v_{i} w_{i+1}\right)\right|$ $=\left|\operatorname{LR}\left(u_{i} v_{i}\right)\right|=\left|\operatorname{LR}\left(v_{i} u_{i+1}\right)\right|=9$ and $\left|\mathrm{LR}\left(e_{i}\right)\right| \leq \mid \mathrm{LR}$ $(x) \mid, \forall x \in E\left(A_{5}\right)$. Furthermore, $\left|\cup_{i=1}^{5} \operatorname{LR}\left(e_{i}\right)\right|=15$ and $\left|\operatorname{LR}(x) \cap \cup_{i=1}^{5} \operatorname{LR}\left(e_{i}\right)\right|=9, \forall x \in E\left(A_{5}\right)$ There exists an upper $\operatorname{LRF} \phi: V\left(A_{5}\right) \longrightarrow[0,1]$ defined as $\phi(v)=$ (1/9), $\forall v \in V\left(A_{5}\right)$. Consequently, by Theorem 1, $\operatorname{dim}_{f l}\left(A_{5}\right) \leq(5 / 3)$. Similarly, for $1 \leq i \leq 5, \quad \mid \mathrm{LR}\left(v_{i}\right.$ $\left.v_{i+1}\right)|=12, \quad| \operatorname{LR}\left(v_{i} v_{i+1}\right)|\geq| \operatorname{LR}(x) \mid, \forall x \in E\left(A_{5}\right)$. Thus, there exist a lower LRF such that $\phi^{\prime}: V\left(A_{6}\right) \longrightarrow[0,1]$ is defined as $\phi^{\prime}(v)=(1 / 12), \forall v \in V\left(A_{5}\right)$. Consequently, by Theorem $2, \operatorname{dim}_{f l}\left(A_{5}\right) \leq(5 / 4)$. Hence, we have

$$
\frac{5}{4} \leq \operatorname{dim}_{f l}\left(A_{5}\right) \leq \frac{5}{3} .
$$

Case 2. For $m>5$, by Lemma 3, $\left|\operatorname{LR}\left(e_{i}\right)\right|=\left|\operatorname{LR}\left(v_{i} w_{i}\right)\right|=$ $\left|\operatorname{LR}\left(v_{i} w_{i+1}\right)\right|=\left|\operatorname{LR}\left(u_{i} v_{i}\right)\right|=\left|\operatorname{LR}\left(v_{i} u_{i+1}\right)\right|=((3 m+3)$

/2) and $\left|\operatorname{LR}\left(e_{i}\right)\right| \leq|\operatorname{LR}(x)|, \forall x \in E\left(A_{5}\right), \forall x \in E\left(A_{m}\right)$. Similarly, $\left|\operatorname{LR}\left(v_{i} v_{i+1}\right)\right|=3 m-3$ and $\left|\operatorname{LR}\left(v_{i} v_{i+1}\right)\right| \geq$ $|\operatorname{LR}(x)|, \forall x \in E\left(A_{m}\right)$. Therefore, $\phi: V\left(A_{m}\right) \longrightarrow[0,1]$ is defined by $\phi(v)=(2 /(3 m+3)), \forall v \in V\left(A_{m}\right)$ is an upper LRF and $\phi^{\prime}: V\left(A_{m}\right) \longrightarrow[0,1]$ is defined by $\phi^{\prime}(v)=(1 /(3 m-3)), \forall v \in V\left(A_{m}\right)$ is a lower LRF. Therefore, by Theorems 1 and 2, we have

$$
\frac{m}{m-1} \leq \operatorname{dim}_{f l}\left(A_{m}\right) \leq \frac{2 m}{m+1} .
$$

Theorem 6. Let $A_{m}$ with $m \geq 6$ be a network of convex polytope, where $m \cong 0(\bmod 2)$. Then,

$$
\frac{m}{m-1} \leq \operatorname{dim}_{f l}\left(A_{m}\right) \leq \frac{6 m}{3 m+2} .
$$

Proof. To prove the result, we have following cases:

Case 1. For $m=6$, the LRN sets are as follows:

$$
\begin{aligned}
& \operatorname{LR}_{1}=\operatorname{LR}\left(u_{1} v_{1}\right)=V\left(A_{6}\right)-\left\{u_{2}, u_{3}, u_{4}, v_{4}, v_{5}, v_{6}, w_{5}, w_{6}\right\}, \\
& \operatorname{LR}_{2}=\operatorname{LR}\left(u_{2} v_{2}\right)=V\left(A_{6}\right)-\left\{u_{2}, u_{3}, u_{4}, v_{5}, v_{6}, v_{1}, w_{6}, w_{1}\right\} \text {, } \\
& \mathrm{LR}_{3}=\operatorname{LR}\left(u_{3} v_{3}\right)=V\left(A_{6}\right)-\left\{u_{3}, u_{4}, u_{5}, v_{6}, v_{1}, v_{2}, w_{1}, w_{2}\right\} \text {, } \\
& \operatorname{LR}_{4}=\operatorname{LR}\left(u_{4} v_{4}\right)=V\left(A_{6}\right)-\left\{u_{4}, u_{5}, u_{6}, v_{1}, v_{2}, v_{3}, w_{2}, w_{3}\right\} \text {, } \\
& \mathrm{LR}_{5}=\operatorname{LR}\left(u_{5} v_{5}\right)=V\left(A_{6}\right)-\left\{u_{5}, u_{6}, u_{1}, v_{2}, v_{3}, v_{4}, w_{3}, w_{4}\right\} \text {, } \\
& \operatorname{LR}_{6}=\operatorname{LR}\left(u_{6} v_{6}\right)=V\left(A_{6}\right)-\left\{u_{6}, u_{1}, u_{2}, v_{3}, v_{4}, v_{5}, w_{4}, w_{5}\right\} \text {, } \\
& \operatorname{LR}_{7}=\operatorname{LR}\left(v_{1} u_{2}\right)=V\left(A_{6}\right)-\left\{u_{1}, u_{5}, u_{6}, v_{2}, v_{3}, v_{4}, w_{3}, w_{4}\right\} \text {, } \\
& \operatorname{LR}_{8}=\operatorname{LR}\left(v_{2} u_{3}\right)=V\left(A_{6}\right)-\left\{u_{2}, u_{6}, u_{1}, v_{3}, v_{4}, v_{5}, w_{4}, w_{5}\right\} \text {, } \\
& \operatorname{LR}_{9}=\operatorname{LR}\left(v_{3} u_{4}\right)=V\left(A_{6}\right)-\left\{u_{3}, u_{1}, u_{2}, v_{4}, v_{5}, v_{1}, w_{5}, w_{6}\right\} \text {, } \\
& \operatorname{LR}_{10}=\operatorname{LR}\left(v_{4} u_{5}\right)=V\left(A_{6}\right)-\left\{u_{4}, u_{2}, u_{3}, v_{5}, v_{6}, v_{1}, w_{6}, w_{1}\right\} \text {, } \\
& \operatorname{LR}_{11}=\operatorname{LR}\left(v_{5} u_{6}\right)=V\left(A_{6}\right)-\left\{u_{5}, u_{3}, u_{4}, v_{6}, v_{1}, v_{2}, w_{1}, w_{2}\right\} \text {, } \\
& \operatorname{LR}_{12}=\operatorname{LR}\left(v_{6} u_{1}\right)=V\left(A_{6}\right)-\left\{u_{6}, u_{4}, u_{5}, v_{1}, v_{5}, w_{3}, w_{2}, w_{3}\right\} \text {, } \\
& \operatorname{LR}_{13}=\operatorname{LR}\left(v_{1} w_{1}\right)=V\left(A_{6}\right)-\left\{u_{5}, u_{6}, v_{4}, v_{5}, v_{6}, w_{2}, w_{3}, w_{4}\right\} \text {, } \\
& \mathrm{LR}_{14}=\operatorname{LR}\left(v_{2} w_{2}\right)=V\left(A_{6}\right)-\left\{u_{6}, u_{1}, v_{5}, v_{6}, v_{1}, w_{3}, w_{4}, w_{5}\right\} \text {, } \\
& \operatorname{LR}_{15}=\operatorname{LR}\left(v_{3} w_{3}\right)=V\left(A_{6}\right)-\left\{u_{1}, u_{2}, v_{6}, v_{1}, v_{2}, w_{4}, w_{5}, w_{6}\right\}, \\
& \mathrm{LR}_{16}=\operatorname{LR}\left(v_{4} w_{4}\right)=V\left(A_{6}\right)-\left\{u_{2}, u_{3}, v_{1}, v_{2}, v_{3}, w_{5}, w_{6}, w_{1}\right\} \text {, } \\
& \mathrm{LR}_{17}=\operatorname{LR}\left(v_{5} w_{5}\right)=V\left(A_{6}\right)-\left\{u_{3}, u_{4}, v_{2}, v_{3}, v_{4}, w_{6}, w_{1}, w_{2}\right\}, \\
& \operatorname{LR}_{18}=\operatorname{LR}\left(v_{6} w_{6}\right)=V\left(A_{6}\right)-\left\{u_{4}, u_{5}, v_{3}, v_{4}, v_{5}, w_{1}, w_{2}, w_{3}\right\} \text {, } \\
& \mathrm{LR}_{19}=\operatorname{LR}\left(v_{1} w_{2}\right)=V\left(A_{6}\right)-\left\{u_{3}, u_{4}, v_{2}, v_{3}, v_{4}, w_{1}, w_{5}, w_{6}\right\} \text {, } \\
& \mathrm{LR}_{20}=\operatorname{LR}\left(v_{2} w_{3}\right)=V\left(A_{6}\right)-\left\{u_{4}, u_{5}, v_{3}, v_{4}, v_{5}, w_{2}, w_{6}, w_{1}\right\} \text {, } \\
& \mathrm{LR}_{21}=\operatorname{LR}\left(v_{3} w_{4}\right)=V\left(A_{6}\right)-\left\{u_{5}, u_{6}, v_{4}, v_{5}, v_{6}, w_{3}, w_{1}, w_{2}\right\} \text {, } \\
& \operatorname{LR}_{22}=\operatorname{LR}\left(v_{4} w_{5}\right)=V\left(A_{6}\right)-\left\{u_{6}, u_{1}, v_{5}, v_{6}, v_{1}, w_{4}, w_{2}, w_{3}\right\} \text {, } \\
& \operatorname{LR}_{23}=\operatorname{LR}\left(v_{5} w_{6}\right)=V\left(A_{6}\right)-\left\{u_{1}, u_{2}, v_{6}, v_{1}, v_{2}, w_{5}, w_{3}, w_{4}\right\}, \\
& \operatorname{LR}_{24}=\operatorname{LR}\left(v_{6} w_{1}\right)=V\left(A_{6}\right)-\left\{u_{2}, u_{3}, v_{1}, v_{2}, v_{3}, w_{6}, w_{4}, w_{5}\right\} \text {, } \\
& \operatorname{LR}_{25}=\operatorname{LR}\left(u_{1} u_{2}\right)=V\left(A_{6}\right)-\left\{v_{1}, v_{4}, w_{1}, w_{2}\right\} \text {, } \\
& \mathrm{LR}_{26}=\operatorname{LR}\left(u_{2} u_{3}\right)=V\left(A_{6}\right)-\left\{v_{2}, v_{5}, w_{2}, w_{3}\right\} \text {, } \\
& \operatorname{LR}_{27}=\operatorname{LR}\left(u_{3} u_{4}\right)=V\left(A_{6}\right)-\left\{v_{3}, v_{6}, w_{3}, w_{4}\right\}, \\
& \operatorname{LR}_{28}=\operatorname{LR}\left(u_{4} u_{5}\right)=V\left(A_{6}\right)-\left\{v_{4}, v_{1}, w_{4}, w_{5}\right\} \text {, } \\
& \mathrm{LR}_{29}=\operatorname{LR}\left(u_{5} u_{6}\right)=V\left(A_{6}\right)-\left\{v_{5}, v_{2}, w_{4}, w_{1}\right\} \text {, } \\
& \operatorname{LR}_{30}=\operatorname{LR}\left(u_{6} u_{1}\right)=V\left(A_{6}\right)-\left\{v_{6}, v_{3}, w_{4}, w_{1}\right\} \text {, } \\
& \mathrm{LR}_{31}=\operatorname{LR}\left(w_{1} w_{2}\right)=V\left(A_{6}\right)-\left\{u_{1}, u_{2}, v_{1}, v_{4}\right\} \text {, } \\
& \mathrm{LR}_{32}=\operatorname{LR}\left(w_{2} w_{3}\right)=V\left(A_{6}\right)-\left\{u_{2}, u_{3}, v_{2}, v_{5}\right\} \text {, } \\
& \mathrm{LR}_{33}=\operatorname{LR}\left(w_{3} w_{4}\right)=V\left(A_{6}\right)-\left\{u_{3}, u_{4}, v_{3}, v_{6}\right\} \text {, } \\
& \mathrm{LR}_{34}=\operatorname{LR}\left(w_{4} w_{5}\right)=V\left(A_{6}\right)-\left\{u_{4}, u_{5}, v_{4}, v_{1}\right\} \text {, } \\
& \mathrm{LR}_{35}=\operatorname{LR}\left(w_{5} w_{6}\right)=V\left(A_{6}\right)-\left\{u_{5}, u_{6}, v_{5}, v_{2}\right\}, \\
& \mathrm{LR}_{36}=\operatorname{LR}\left(w_{6} w_{1}\right)=V\left(A_{6}\right)-\left\{u_{6}, u_{1}, v_{6}, v_{3}\right\} \text {, } \\
& \operatorname{LR}_{37}=\operatorname{LR}\left(v_{1} v_{2}\right)=V\left(A_{6}\right)-\left\{u_{2}, w_{2}, w_{5}\right\}, \\
& \operatorname{LR}_{38}=\operatorname{LR}\left(v_{2} v_{3}\right)=V\left(A_{6}\right)-\left\{u_{3}, w_{3}, w_{6}\right\}, \\
& \mathrm{LR}_{39}=\operatorname{LR}\left(v_{3} v_{4}\right)=V\left(A_{6}\right)-\left\{u_{4}, w_{4}, w_{1}\right\}, \\
& \mathrm{LR}_{40}=\operatorname{LR}\left(v_{4} v_{5}\right)=V\left(A_{6}\right)-\left\{u_{5}, w_{5}, w_{2}\right\}, \\
& \operatorname{LR}_{41}=\operatorname{LR}\left(v_{5} v_{6}\right)=V\left(A_{6}\right)-\left\{u_{6}, w_{6}, w_{3}\right\} \text {, } \\
& \operatorname{LR}_{42}=\operatorname{LR}\left(v_{6} v_{1}\right)=V\left(A_{6}\right)-\left\{u_{1}, w_{1}, w_{4}\right\} .
\end{aligned}
$$

For $1 \leq i \leq 6, \quad\left|\operatorname{LR}\left(e_{i}\right)\right|=\left|\operatorname{LR}\left(v_{i} w_{i}\right)\right|=\left|\operatorname{LR}\left(v_{i} w_{i+1}\right)\right|=$ $\left|\operatorname{LR}\left(u_{i} v_{i}\right)\right|=\left|\operatorname{LR}\left(v_{i} u_{i+1}\right)\right|=10$ and $\left|\operatorname{LR}\left(e_{i}\right)\right| \leq|\operatorname{LR}(x)|$, $\forall x \in E\left(A_{5}\right)$. Furthermore, $\left|\cup_{i=1}^{6} \operatorname{LR}\left(e_{i}\right)\right|=18$ and 
$\left|\operatorname{LR}(x) \cap \cup_{i=1}^{6} \operatorname{LR}\left(e_{i}\right)\right|=10, \forall x \in E\left(A_{6}\right)$ There exists an upper $\operatorname{LRF} \phi: V\left(A_{6}\right) \longrightarrow[0,1]$ defined as $\phi(v)=$ $(1 / 10), \forall v \in V\left(A_{6}\right)$. Consequently, by Theorem 1, $\operatorname{dim}_{f l}\left(A_{5}\right) \leq(9 / 5)$. Similarly, for $1 \leq i \leq 5, \quad \mid \operatorname{LR}\left(v_{i}\right.$ $\left.v_{i+1}\right) \mid=15$ and $\left|\operatorname{LR}\left(v_{i} v_{i+1}\right)\right| \geq|\operatorname{LR}(x)|, \forall x \in E\left(A_{6}\right)$. Thus, there exists a lower LRF such that $\phi^{\prime}$ : $V\left(A_{6}\right) \longrightarrow[0,1]$ is defined as $\phi^{\prime}(v)=(1 / 15)$, $\forall v \in V\left(A_{6}\right)$. Consequently, by Theorem 2, $\operatorname{dim}_{f l}\left(A_{6}\right) \geq(6 / 5)$. Hence, we have

$$
\frac{6}{5} \leq \operatorname{dim}_{f l}\left(A_{5}\right) \leq \frac{9}{5}
$$

Case 2. For $m>6$, by Lemma 4 , $\left|\operatorname{LR}\left(e_{i}\right)\right|=\left|\operatorname{LR}\left(u_{i} v_{i}\right)\right|=$ $\left|\operatorname{LR}\left(v_{i} w_{i}\right)\right|=\left|\operatorname{LR}\left(v_{i} w_{i+1}\right)\right|=\left|\operatorname{LR}\left(v_{i} u_{i+1}\right)\right|=((3 m+2) /$

2) and $\left|\operatorname{LR}\left(e_{i}\right)\right| \leq|\operatorname{LR}(x)|, \quad \forall x \in E\left(A_{m}\right)$. Similarly, $\left|\operatorname{LR}\left(v_{i} v_{i+1}\right)\right|=3 m-3$ and $\left|\operatorname{LR}\left(v_{i} v_{i+1}\right)\right| \geq|\operatorname{LR}(x)|, \forall x \in$ $E\left(A_{m}\right)$. Therefore, $\phi: V\left(A_{m}\right) \longrightarrow[0,1]$ is defined by $\phi(v)=(2 /(3 m+2)), \forall v \in V\left(A_{m}\right)$ is an upper LRF and $\phi^{\prime}: V\left(A_{m}\right) \longrightarrow[0,1]$ is defined by $\phi^{\prime}(v)=(1 /(3 m$ $-3)), \forall v \in V\left(A_{m}\right)$ is a lower LRF. Therefore, by Theorems 1 and 2 , we have

$$
\frac{m}{m-1} \leq \operatorname{dim}_{f l}\left(A_{m}\right) \leq \frac{6 m}{3 m+2} .
$$

3.3. LRN Sets and LFMD of Convex Polytope $S_{m}$. This subsection deals with the results for the LRN sets and LFMD of the convex polytopes network $S_{m}$ with $m \geq 5$.

Lemma 5. Let $S_{m}$ be a network of convex polytope, where $m \geq 5$ and $m \cong 1(\bmod 2)$. Then, for $1 \leq i \leq m$,
(a) $\left|\operatorname{LR}\left(e_{i}\right)\right|=\left|\operatorname{LR}\left(v_{i} w_{i}\right)\right|=\left|\operatorname{LR}\left(w_{i} v_{i+1}\right)\right|=2(m+1)$ and $\left|\operatorname{LR}\left(e_{i}^{\prime}\right)\right|=\left|\operatorname{LR}\left(u_{i} v_{i}\right)\right|=\left|\operatorname{LR}\left(v_{i} w_{i}\right)\right|=4 m$ with $\mid \cup_{i=1}^{m}$ $\operatorname{LR}\left(e_{i}\right) \mid=4 m$
(b) $\left|L R\left(e_{i}\right)\right| \leq|L R(x)| \leq\left|L R\left(e_{i}^{\prime}\right)\right|, \forall x \in E\left(S_{m}\right)$
(c) $\left|\operatorname{LR}(x) \cap \cup_{i=1}^{m} \operatorname{LR}\left(e_{i}\right)\right| \geq\left|\operatorname{LR}\left(e_{i}\right)\right|, \forall x \in E\left(S_{m}\right)$

Proof. Assume that $u_{i}, v_{i}, w_{i}$, and $z_{i}$ are inner, middle, and outer vertices of $S_{m}$, respectively, where $1 \leq i \leq m$ and $m+1 \cong 1(\bmod m)$

(a) Consider $\operatorname{LR}\left(v_{i} w_{i}\right)=\left\{u_{i}, u_{((m+2 i+1) / 2)}, u_{((m+2 i+1) / 2)+1}\right.$, $\ldots, u_{m}, \quad v_{i}, v_{((m+2 i+1) / 2)}, v_{((m+2 i+1) / 2)+1}, \ldots, v_{m}, w_{i}$, $w_{((m+2 i+1) / 2)}, w_{((m+2 i+1) / 2)+1}, \ldots, w_{m}, z_{i}, z_{i+1}, z_{i+2}, \ldots$, $\left.z_{((m+2 i+1) / 2)+1}\right\}, \quad \operatorname{LR}\left(w_{i} v_{i+1}\right)=\left\{u_{i+1}, u_{((m+2 i+1) / 2)}\right.$, $u_{((m+2 i+1) / 2)+1}, \ldots, u_{m}, v_{i+1}, v_{i+2}, \quad v_{i+3}, \ldots$, $v_{((m+2 i+1) / 2)}, w_{i}, w_{((m+2 i+1) / 2)}, w_{((m+2 i+3) / 2)}, \ldots, w_{m}, z_{i}$, $\left.z_{((m+2 i+1) / 2)}, z_{((m+2 i+3) / 2)}, \ldots, z_{m}\right\}, \quad$ and $\operatorname{LR}\left(u_{i} v_{i}\right)=$ $\operatorname{LR}\left(w_{i} z_{i}\right)=V\left(S_{m}\right)$. Therefore, $\left|\operatorname{LR}\left(e_{i}\right)\right|=2(m+1)$ and $\left|\operatorname{LR}\left(e_{i}^{\prime}\right)\right|=4 m$. Also, $\left|\cup_{i=1}^{m} \operatorname{LR}\left(e_{i}\right)\right|=\left|V\left(S_{m}\right)\right|=$ $4 m$.

(b) The LRN sets of $S_{m}$ other than $\operatorname{LR}\left(v_{i} w_{i}\right), \operatorname{LR}\left(w_{i} v_{i+1}\right)$, $\operatorname{LR}\left(u_{i} v_{i}\right)$, and $\operatorname{LR}\left(w_{i} z_{i}\right)$ are $\operatorname{LR}\left(v_{i} v_{i+1}\right)=V\left(S_{m}\right)-$ $\left\{v_{((m+2 i+1) / 2)}, u_{((m+2 i+1) / 2)}, w_{i}, z_{i}\right\}, \quad \operatorname{LR}\left(u_{i} u_{i+1}\right)=V$ $\left(S_{m}\right)-\quad\left\{u_{((m+2 i+1) / 2)}, v_{((m+2 i+1) / 2)}, w_{i}, z_{i}\right\}, \quad \operatorname{LR}\left(w_{i}\right.$ $\left.w_{i+1}\right)=V\left(S_{m}\right)-\left\{w_{((m+2 i+1) / 2)}, z_{((m+2 i+1) / 2)}, u_{i+1}, v_{i+1}\right\}$, and $\operatorname{LR}\left(z_{i} z_{i+1}\right)=V\left(S_{m}\right)-\left\{u_{i+1}, v_{i+1}, w_{((m+2 i+1) / 2)}\right.$, $\left.z_{((m+2 i+1) / 2)}\right\}$.

The cardinalities of these LRN sets are obtained as given in Table 5.

From Table 5, it is clear that $\left|\operatorname{LR}\left(e_{i}\right)\right| \leq|\operatorname{LR}(x)| \leq$ $\left|\operatorname{LR}\left(e_{i}^{\prime}\right)\right|, \forall x \in E\left(S_{m}\right)$.

(c) Since $\left|\cup_{i=1}^{m} \operatorname{LR}\left(e_{i}\right)\right|=\left|V\left(S_{m}\right)\right|$, therefore $\mid \operatorname{LR}(x) \cap$ $\cup_{i=1}^{m} \operatorname{LR}\left(e_{i}\right)|=| \operatorname{LR}(x)|\geq| \operatorname{LR}\left(e_{i}\right) \mid$.

Lemma 6. Let $S_{m}$ be a network of convex polytope, where $m \geq 6$ and $m \cong 0(\bmod 2)$. Then, for $1 \leq i \leq m$,

(a) $\left|\operatorname{LR}\left(e_{i}\right)\right|=\left|\operatorname{LR}\left(v_{i} w_{i}\right)\right|=\left|\operatorname{LR}\left(w_{i} v_{i+1}\right)\right|=2 m, \quad\left|\operatorname{LR}\left(e_{i}^{\prime}\right)\right|$ $=\left|\operatorname{LR}\left(w_{i} z_{i}\right)\right|=\left|\operatorname{LR}\left(u_{i} v_{i}\right)\right|=4 m$, and $\left|\cup_{i=1}^{m} \operatorname{LR}\left(e_{i}\right)\right|=$ $4 m$

(b) $\left|L R\left(v_{i} w_{i}\right)\right| \leq|L R(x)| \leq\left|L R\left(e_{i}^{\prime}\right)\right|, \forall x \in E\left(S_{m}\right)$

(c) $\left|L R(x) \cap \cup_{i=1}^{m} L R\left(e_{i}\right)\right| \geq\left|L R\left(e_{i}\right)\right|, \forall x \in E\left(S_{m}\right)$

Proof. Assume that $u_{i}, v_{i}, w_{i}$, and $z_{i}$ are inner, middle and outer vertices of $S_{m}$, respectively, where $1 \leq i \leq m$ and $m+1 \cong 1(\bmod m)$.

(a) Consider $\operatorname{LR}\left(v_{i} w_{i}\right)=\left\{u_{i}, u_{((m+2 i+2) / 2)}, u_{((m+2 i+2) / 2)+1}\right.$, $\ldots, u_{m}, \quad v_{i}, v_{((m+2 i+2) / 2)}, v_{((m+2 i+2) / 2)+1}, \ldots, v_{m}, w_{i}$, $\left.w_{i+1}, w_{i+2}, \ldots, w_{(m / 2)}, z_{i}, z_{i+1}, z_{i+2}, \ldots, z_{(m / 2)}\right\}, \operatorname{LR}\left(w_{i}\right.$ $\left.v_{i+1}\right)=\left\{u_{i}, u_{i+1}, u_{i+2}, . ., u_{((m+2 i) / 2)}, v_{i+1}, v_{i+2}, v_{i+3}, \ldots\right.$, $v_{((m+2 i) / 2)}, \quad w_{i}, w_{((m+2 i+2) / 2)}, w_{((m+2 i+3) / 2)}, \ldots, w_{m}, z_{i}$, $\left.z_{((m+2 i+2) / 2)}, z_{((m+2 i+3) / 2)}, \ldots, z_{m}\right\}$, and $\operatorname{LR}\left(w_{i} z_{i}\right)=$ $\operatorname{LR}\left(u_{i} v_{i}\right)=V\left(S_{m}\right)$. Therefore, $\left|\operatorname{LR}\left(e_{i}\right)\right|=2 m$ and $\left|\operatorname{LR}\left(e_{i}^{\prime}\right)\right|=4 m$. Also, $\left|\cup_{i=1}^{m} \operatorname{LR}\left(e_{i}\right)\right|=4 m$.

(b) The LRN sets other than $\operatorname{LR}\left(v_{i} w_{i}\right), \operatorname{LR}\left(w_{i} v_{i+1}\right)$, $\operatorname{LR}\left(u_{i} v_{i}\right)$, and $\operatorname{LR}\left(w_{i} z_{i}\right)$ are $\operatorname{LR}\left(v_{i} v_{i+1}\right)=V\left(S_{m}\right)$ $-\left\{w_{i}, w_{((m+2 i) / 2)}\right\}, \quad \operatorname{LR}\left(w_{i} w_{i+1}\right)=V\left(S_{m}\right)-\left\{u_{i+1}\right.$, $\left.u_{((m+2 i+2) / 2)}, v_{i+1}, v_{((m+2 i+2) / 2)}\right\}, \quad \operatorname{LR}\left(u_{i} u_{i+1}\right)=V\left(S_{m}\right)$ $-\left\{w_{i}, z_{i}, w_{((m+2 i) / 2)}, z_{((m+2 i) / 2)}\right\}$, and $\operatorname{LR}\left(z_{i} z_{i+1}\right)=V$ $\left(S_{m}\right)-\left\{u_{i+1}, u_{((m+2 i+2) / 2)}, v_{i+1}, v_{((m+2 i+2) / 2)}\right\}$.

The cardinalities of these LRN sets are obtained as given in Table 6.

From Table 6 , it is clear that $\left|\operatorname{LR}\left(e_{i}\right)\right| \leq|\operatorname{LR}(x)| \leq$ $\left|\operatorname{LR}\left(e_{i}^{\prime}\right)\right|, \forall x \in E\left(S_{m}\right)$.

(c) Since $\left|\cup_{i=1}^{m} \operatorname{LR}\left(e_{i}\right)\right|=\left|V\left(S_{m}\right)\right|=4 m$, therefore $\mid \mathrm{LR}$ $(x) \cap \cup_{i=1}^{m} \operatorname{LR}\left(e_{i}\right)|=| \operatorname{LR}(x)|\geq| \operatorname{LR}\left(e_{i}\right) \mid$.

Theorem 7. Let $S_{m}$ with $m \geq 5$ be a network of convex polytope, where $m \cong 1(\bmod 2)$. Then,

$$
1<\operatorname{dim}_{f l}\left(S_{m}\right) \leq \frac{2 m}{m+1} .
$$

Proof. To prove the result, we have the following cases:

Case 1. For $m=5$, the LRN sets are as follows: 
TABLE 5: Cardinalities of LRN sets of $S_{m}$ for $m \cong 1(\bmod 2)$.

\begin{tabular}{lc}
\hline Cardinalities of LRN sets & Comparison \\
\hline$\left|\operatorname{LR}\left(v_{i} v_{i+1}\right)\right|=4 m-4$ & $4 m>4 m-4>2(m+1)$ \\
$\left|\operatorname{LR}\left(u_{i} u_{i+1}\right)\right|=4 m-4$ & $4 m>4 m-4>2(m+1)$ \\
$\left|\operatorname{LR}\left(w_{i} w_{i+1}\right)\right|=4 m-4$ & $4 m>4 m-4>2(m+1)$ \\
$\left|\operatorname{LR}\left(z_{i} z_{i+1}\right)\right|=4 m-4$ & $4 m>4 m-4>2(m+1)$ \\
\hline
\end{tabular}

TABLE 6: Cardinalities of LRN sets of $S_{m}$ for $m \cong 0(\bmod 2)$.

\begin{tabular}{lc}
\hline Cardinalities of LRN sets & Comparison \\
\hline$\left|\operatorname{LR}\left(v_{i} v_{i+1}\right)\right|=4 m-2$ & $4 m>4 m-2>2 m$ \\
$\left|\operatorname{LR}\left(u_{i} u_{i+1}\right)\right|=4 m-4$ & $4 m>4 m-4>2 m$ \\
$\left|\operatorname{LR}\left(w_{i} w_{i+1}\right)\right|=4 m-4$ & $4 m>4 m-4>2 m$ \\
$\left|\operatorname{LR}\left(z_{i} z_{i+1}\right)\right|=4 m-4$ & $4 m>4 m-4>2 m$ \\
\hline
\end{tabular}

$$
\begin{aligned}
& \mathrm{LR}_{1}=\operatorname{LR}\left(v_{1} w_{1}\right)=V\left(S_{5}\right)-\left\{u_{2}, u_{3}, v_{2}, v_{3}, w_{5}, w_{4}, z_{4}, z_{5}\right\}, \\
& \operatorname{LR}_{2}=\operatorname{LR}\left(v_{2} w_{2}\right)=V\left(S_{5}\right)-\left\{u_{3}, u_{4}, v_{3}, v_{4}, w_{1}, w_{5}, z_{5}, z_{1}\right\}, \\
& \mathrm{LR}_{3}=\operatorname{LR}\left(v_{3} w_{3}\right)=V\left(S_{5}\right)-\left\{u_{4}, u_{5}, v_{4}, v_{5}, w_{2}, w_{1}, z_{1}, z_{2}\right\} \text {, } \\
& \operatorname{LR}_{4}=\operatorname{LR}\left(v_{4} w_{4}\right)=V\left(S_{5}\right)-\left\{u_{5}, u_{1}, v_{5}, v_{1}, w_{3}, w_{2}, z_{2}, z_{3}\right\} \text {, } \\
& \operatorname{LR}_{5}=\operatorname{LR}\left(v_{5} w_{5}\right)=V\left(S_{5}\right)-\left\{u_{1}, u_{2}, v_{1}, v_{2}, w_{4}, w_{3}, z_{3}, z_{4}\right\} \text {, } \\
& \operatorname{LR}_{6}=\operatorname{LR}\left(v_{2} w_{1}\right)=V\left(S_{5}\right)-\left\{u_{1}, u_{5}, v_{1}, v_{5}, w_{2}, w_{3}, z_{2}, z_{3}\right\} \text {, } \\
& \operatorname{LR}_{7}=\operatorname{LR}\left(v_{3} w_{2}\right)=V\left(S_{5}\right)-\left\{u_{2}, u_{1}, v_{2}, v_{1}, w_{3}, w_{4}, z_{3}, z_{4}\right\} \text {, } \\
& \operatorname{LR}_{8}=\operatorname{LR}\left(v_{4} w_{3}\right)=V\left(S_{5}\right)-\left\{u_{3}, u_{2}, v_{3}, v_{2}, w_{4}, w_{5}, z_{4}, z_{5}\right\} \text {, } \\
& \mathrm{LR}_{9}=\operatorname{LR}\left(v_{5} w_{4}\right)=V\left(S_{5}\right)-\left\{u_{4}, u_{3}, v_{4}, v_{3}, w_{5}, w_{1}, z_{5}, z_{1}\right\} \text {, } \\
& \operatorname{LR}_{10}=\operatorname{LR}\left(v_{1} w_{5}\right)=V\left(S_{5}\right)-\left\{u_{5}, u_{4}, v_{5}, v_{4}, w_{1}, w_{2}, z_{1}, z_{2}\right\} \text {, } \\
& \operatorname{LR}_{11}=\operatorname{LR}\left(z_{1} z_{2}\right)=V\left(S_{5}\right)-\left\{v_{2}, u_{2}, u_{5}, w_{4}, z_{4}\right\} \text {, } \\
& \operatorname{LR}_{12}=\operatorname{LR}\left(z_{2} z_{3}\right)=V\left(S_{5}\right)-\left\{v_{3}, u_{3}, u_{2}, w_{5}, z_{5}\right\} \text {, } \\
& \operatorname{LR}_{13}=\operatorname{LR}\left(z_{3} z_{4}\right)=V\left(S_{5}\right)-\left\{v_{4}, u_{4}, u_{3}, w_{2}, z_{2}\right\} \text {, } \\
& \operatorname{LR}_{14}=\operatorname{LR}\left(z_{4} z_{5}\right)=V\left(S_{5}\right)-\left\{v_{5}, u_{5}, u_{4}, w_{3}, z_{3}\right\} \text {, } \\
& \operatorname{LR}_{15}=\operatorname{LR}\left(z_{5} z_{1}\right)=V\left(S_{5}\right)-\left\{v_{1}, u_{1}, u_{5}, w_{4}, z_{4}\right\} \text {, } \\
& \operatorname{LR}_{16}=\operatorname{LR}\left(w_{1} z_{1}\right)=V\left(S_{5}\right)-\left\{w_{2}, w_{3}, w_{5}\right\}, \\
& \operatorname{LR}_{17}=\operatorname{LR}\left(w_{2} z_{2}\right)=V\left(S_{5}\right)-\left\{w_{3}, w_{4}, w_{1}\right\}, \\
& \operatorname{LR}_{18}=\operatorname{LR}\left(w_{3} z_{3}\right)=V\left(S_{5}\right)-\left\{w_{4}, w_{5}, w_{2}\right\}, \\
& \operatorname{LR}_{19}=\operatorname{LR}\left(w_{4} z_{4}\right)=V\left(S_{5}\right)-\left\{w_{5}, w_{1}, w_{3}\right\} \text {, } \\
& \operatorname{LR}_{20}=\operatorname{LR}\left(w_{5} z_{5}\right)=V\left(S_{5}\right)-\left\{w_{1}, w_{2}, w_{4}\right\}, \\
& \operatorname{LR}_{21}=\operatorname{LR}\left(v_{1} v_{2}\right)=V\left(S_{5}\right)-\left\{v_{4}, w_{1}, z_{1}\right\}, \\
& \operatorname{LR}_{22}=\operatorname{LR}\left(v_{2} v_{3}\right)=V\left(S_{5}\right)-\left\{v_{5}, w_{2}, z_{2}\right\}, \\
& \operatorname{LR}_{23}=\operatorname{LR}\left(v_{3} v_{4}\right)=V\left(S_{5}\right)-\left\{v_{1}, w_{3}, z_{3}\right\} \text {, } \\
& \mathrm{LR}_{24}=\operatorname{LR}\left(v_{4} v_{5}\right)=V\left(S_{5}\right)-\left\{v_{2}, w_{4}, z_{4}\right\}, \\
& \operatorname{LR}_{25}=\operatorname{LR}\left(v_{5} v_{1}\right)=V\left(S_{5}\right)-\left\{v_{3}, w_{5}, z_{5}\right\} \text {, } \\
& \mathrm{LR}_{26}=\operatorname{LR}\left(u_{1} u_{2}\right)=V\left(S_{5}\right)-\left\{u_{4}, w_{1}, z_{1}\right\}, \\
& \operatorname{LR}_{27}=\operatorname{LR}\left(u_{2} u_{3}\right)=V\left(S_{5}\right)-\left\{u_{5}, w_{2}, z_{2}\right\} \text {, } \\
& \mathrm{LR}_{28}=\operatorname{LR}\left(u_{3} u_{4}\right)=V\left(S_{5}\right)-\left\{u_{1}, w_{3}, z_{3}\right\} \text {, } \\
& \operatorname{LR}_{29}=\operatorname{LR}\left(u_{4} u_{5}\right)=V\left(S_{5}\right)-\left\{u_{2}, w_{4}, z_{4}\right\} \text {, } \\
& \operatorname{LR}_{30}=\operatorname{LR}\left(u_{5} u_{1}\right)=V\left(S_{5}\right)-\left\{u_{3}, w_{4}, z_{5}\right\} \text {, }
\end{aligned}
$$

$$
\begin{aligned}
& \mathrm{LR}_{31}=\operatorname{LR}\left(u_{1} v_{1}\right)=V\left(S_{5}\right), \\
& \operatorname{LR}_{32}=\operatorname{LR}\left(u_{2} v_{2}\right)=V\left(S_{5}\right), \\
& \mathrm{LR}_{33}=\operatorname{LR}\left(u_{3} v_{3}\right)=V\left(S_{5}\right), \\
& \operatorname{LR}_{34}=\operatorname{LR}\left(u_{4} v_{4}\right)=V\left(S_{5}\right), \\
& \operatorname{LR}_{35}=\operatorname{LR}\left(u_{5} v_{5}\right)=V\left(S_{5}\right) .
\end{aligned}
$$

For $1 \leq i \leq 5,\left|\operatorname{LR}\left(e_{i}\right)\right|=\left|\operatorname{LR}\left(v_{i} w_{i}\right)\right|=\left|\operatorname{LR}\left(v_{i} w_{i+1}\right)\right|=12$ and $\left|\operatorname{LR}\left(e_{i}\right)\right| \leq|\operatorname{LR}(x)|, \forall x \in E\left(S_{5}\right)$. Furthermore, $\left|\cup_{i=1}^{5} \operatorname{LR}\left(e_{i}\right)\right|=20 \quad$ and $\left|\operatorname{LR}(x) \cap \cup_{i=1}^{5} \operatorname{LR}\left(e_{i}\right)\right|=9$, $\forall x \in E\left(S_{5}\right)$. There exists an upper LRF $\phi: V$ $\left(S_{5}\right) \longrightarrow[0,1]$ defined as $\phi(v)=(1 / 12), \forall v \in V\left(S_{5}\right)$. Consequently, by Theorem $1, \operatorname{dim}_{f l}\left(S_{5}\right) \leq(5 / 3)$. Similarly, for $1 \leq i \leq 5, \quad\left|\operatorname{LR}\left(u_{i} v_{i}\right)\right|=24$ and $\mid \operatorname{LR}\left(u_{i}\right.$ $\left.v_{i}\right)|\geq| \operatorname{LR}(x) \mid, \forall x \in E\left(S_{5}\right)$. Thus, there exist a lower LRF such that $\phi^{\prime}: V\left(S_{5}\right) \longrightarrow[0,1]$ is defined as $\phi^{\prime}(v)=(1 / 24), \forall v \in V\left(S_{5}\right)$. Consequently, by Theorem 2 , $\operatorname{dim}_{f l}\left(S_{5}\right) \geq 1$. Since $S_{5}$ is nonbipartite network, therefore $\operatorname{dim}_{f l}\left(S_{5}\right)$ must be greater than 1 . Hence, we have

$$
1<\operatorname{dim}_{f l}\left(S_{5}\right) \leq \frac{5}{3} .
$$

Case 2. For $m>5$, by Lemma 5, $\left|\operatorname{LR}\left(e_{i}\right)\right|=\left|\operatorname{LR}\left(v_{i} w_{i}\right)\right|=$ $\left|\operatorname{LR}\left(v_{i} w_{i+1}\right)\right|=2(m+1)$ and $\left|\operatorname{LR}\left(e_{i}\right)\right| \leq|\operatorname{LR}(x)|, \forall x \in E$ $\left(S_{m}\right)$. Similarly, $\left|\operatorname{LR}\left(u_{i} v_{i}\right)\right|=4 m$ and $\left|\operatorname{LR}\left(u_{i} v_{i}\right)\right| \geq$ $|\operatorname{LR}(x)|, \forall x \in E\left(S_{m}\right)$. Therefore, $\phi: V\left(S_{m}\right) \longrightarrow[0,1]$ is defined by $\phi(v)=(1 / 2(m+1)), \forall v \in V\left(S_{m}\right)$ is an upper LRF and $\phi^{\prime}: V\left(S_{m}\right) \longrightarrow[0,1]$ is defined by $\phi^{\prime}(v)=(1 / 4 m), \forall v \in V\left(A_{m}\right)$ is a lower LRF. Therefore, by Theorems 1 and 2 , we have

$$
1<\operatorname{dim}_{f l}\left(S_{m}\right) \leq \frac{2 m}{m+1} .
$$

Theorem 8. Let $S_{m}$ with $m \geq 6$ be a network of convex polytope, where $m \cong 0(\bmod 2)$. Then,

$$
1<\operatorname{dim}_{f l}\left(S_{m}\right) \leq 2 \text {. }
$$

Proof. To prove the result, we have following cases:

Case 1. For $m=6$, the LRN sets are as follows:

$$
\begin{aligned}
& \operatorname{LR}_{1}=\operatorname{LR}\left(u_{1} u_{2}\right)=V\left(S_{6}\right)-\left\{w_{1}, w_{4}, z_{1}, z_{4}\right\}, \\
& \operatorname{LR}_{2}=\operatorname{LR}\left(u_{2} u_{3}\right)=V\left(S_{6}\right)-\left\{w_{2}, w_{5}, z_{2}, z_{5}\right\}, \\
& \operatorname{LR}_{3}=\operatorname{LR}\left(u_{3} u_{4}\right)=V\left(S_{6}\right)-\left\{w_{3}, w_{6}, z_{3}, z_{6}\right\}, \\
& \operatorname{LR}_{4}=\operatorname{LR}\left(u_{4} u_{5}\right)=V\left(S_{6}\right)-\left\{w_{4}, w_{1}, z_{4}, z_{1}\right\}, \\
& \operatorname{LR}_{5}=\operatorname{LR}\left(u_{5} u_{6}\right)=V\left(S_{6}\right)-\left\{w_{5}, w_{2}, z_{5}, z_{2}\right\}, \\
& \operatorname{LR}_{6}=\operatorname{LR}\left(u_{6} u_{1}\right)=V\left(S_{6}\right)-\left\{w_{6}, w_{3}, z_{6}, z_{3}\right\}, \\
& \operatorname{LR}_{7}=\operatorname{LR}\left(w_{1} w_{2}\right)=V\left(S_{6}\right)-\left\{v_{2}, v_{5}, u_{2}, u_{5}\right\},
\end{aligned}
$$




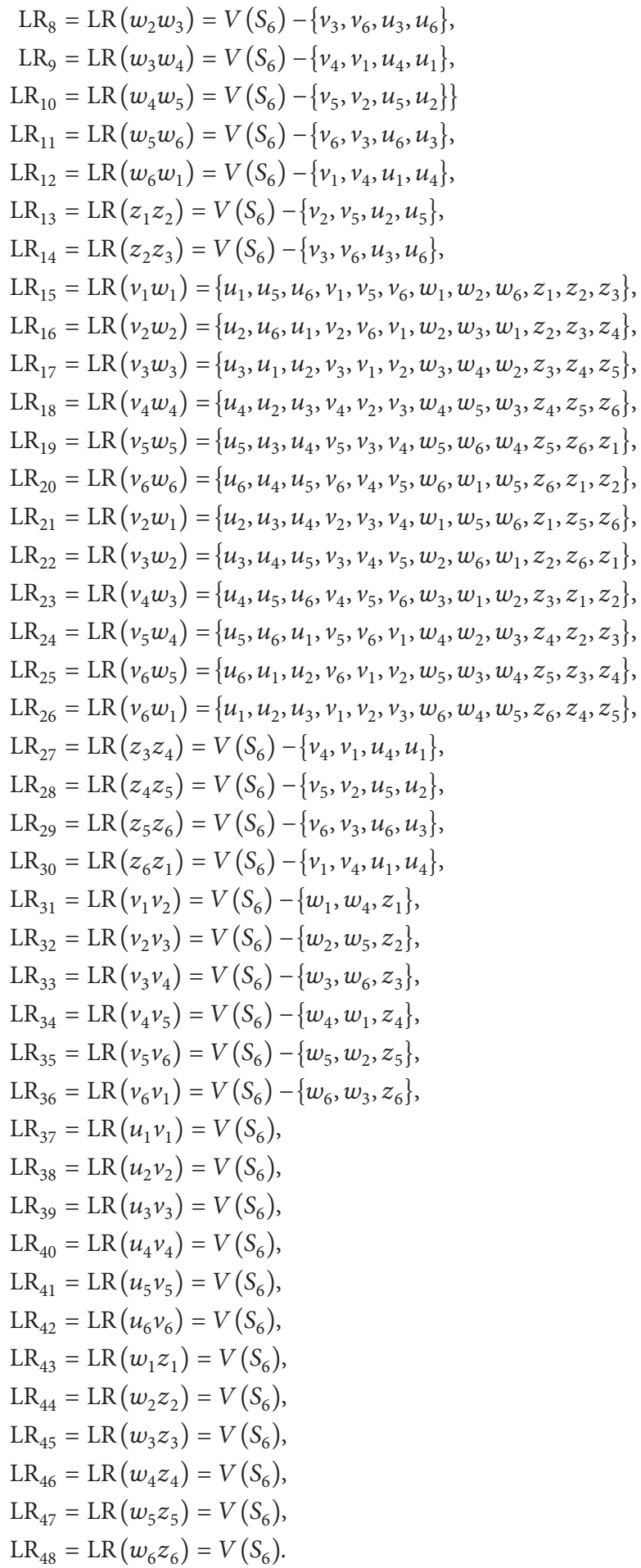

For $1 \leq i \leq 6,\left|\operatorname{LR}\left(e_{i}\right)\right|=\left|\operatorname{LR}\left(v_{i} w_{i}\right)\right|=\left|\operatorname{LR}\left(v_{i} w_{i+1}\right)\right|=12$ and $\left|\operatorname{LR}\left(e_{i}\right)\right| \leq|\operatorname{LR}(x)|, \forall x \in E\left(S_{6}\right)$. Furthermore, $\left|\cup_{i=1}^{6} \operatorname{LR}\left(e_{i}\right)\right|=\left|V\left(S_{6}\right)\right|=20$ and $\mid \operatorname{LR}(x) \cap \cup_{i=1}^{6} \mathrm{LR}$ $\left(e_{i}\right) \mid=12, \forall x \in E\left(S_{6}\right)$. There exists an upper LRF $\phi: V\left(S_{6}\right) \longrightarrow[0,1]$ defined as $\phi(v)=(1 / 12), \forall v \in$ $V\left(S_{6}\right)$. Consequently, by Theorem $1, \operatorname{dim}_{f l}\left(S_{6}\right) \leq 2$. Similarly, for $1 \leq i \leq 6, \quad\left|\operatorname{LR}\left(u_{i} v_{i}\right)\right|=24$ and $\mid \mathrm{LR}$ $\left(u_{i} v_{i}\right)|\geq| \operatorname{LR}(x) \mid, \forall x \in E\left(S_{6}\right)$. Thus, there exists a lower LRF such that $\phi^{\prime}: V\left(S_{6}\right) \longrightarrow[0,1]$ is defined as $\phi^{\prime}(v)=(1 / 24), \forall v \in V\left(S_{5} 6\right)$. Consequently, by Theorem $2, \operatorname{dim}_{f l}\left(S_{6}\right) \geq 1$. Since $S_{6}$ is nonbipartite network, therefore $\operatorname{dim}_{f l}\left(S_{6}\right)$ must be greater than 1 . Hence, we have

$$
1<\operatorname{dim}_{f l}\left(S_{6}\right) \leq 2
$$

Case 2. For $m>6$, by Lemma 6, $\left|\operatorname{LR}\left(e_{i}\right)\right|=\left|\operatorname{LR}\left(v_{i} w_{i}\right)\right|=$ $\left|\operatorname{LR}\left(v_{i} w_{i+1}\right)\right|=2 m$ and $\left|\operatorname{LR}\left(e_{i}\right)\right| \leq|\operatorname{LR}(x)|, \forall x \in E\left(S_{m}\right)$. Similarly, $\left|\operatorname{LR}\left(u_{i} v_{i}\right)\right|=4 m$ and $\left|\operatorname{LR}\left(u_{i} v_{i}\right)\right| \geq|\operatorname{LR}(x)|$, $\forall x \in E\left(S_{m}\right)$. Therefore, $\phi: V\left(S_{m}\right) \longrightarrow[0,1]$ is defined by $\phi(v)=(1 / 2 m), \forall v \in V\left(S_{m}\right)$ is an upper LRF and $\phi^{\prime}: V\left(S_{m}\right) \longrightarrow[0,1]$ is defined by $\phi^{\prime}(v)=(1 / 4 m)$, $\forall v \in V\left(A_{m}\right)$ is a lower LRF. Moreover, $S_{m}$ is a nonbipartite network so $\operatorname{dim}_{f l}\left(S_{m}\right)$ must be greater than 1 . Therefore, by Theorems 1 and 2, we have

$$
1<\operatorname{dim}_{f l}\left(S_{m}\right) \leq 2 \text {. }
$$

3.4. LRN Sets and LFMD of Convex Polytope $Q_{m}$. This subsection deals with the results for the LRN sets and LFMD of the convex polytopes network $Q_{m}$ with $m \geq 5$.

Lemma 7. Let $Q_{m}$ be a network of convex polytope, where $m \geq 5$ and $m \cong 1(\bmod 2)$. Then, for $1 \leq i \leq m$,

(a) $\left|\operatorname{LR}\left(e_{i}\right)\right|=\left|\operatorname{LR}\left(u_{i} u_{i+1}\right)\right|=\left|\operatorname{LR}\left(z_{i} z_{i+1}\right)\right|=\left|\operatorname{LR}\left(u_{i} v_{i}\right)\right|=$ $\left|L R\left(v_{i} w_{i}\right)\right|=\left|L R\left(w_{i} z_{i}\right)\right|=4 m-4$ with $\left|\cup_{i=1}^{m} L R\left(e_{i}\right)\right|=$ $4 m$.

Proof. Assume that $u_{i}, v_{i}$, and $w_{i}$ are inner and middle vertices, respectively, and $z_{i}$ are outer vertices, where $1 \leq i \leq m$ and $m+1 \cong 1(\bmod m)$ :

(a) $\operatorname{LR}\left(u_{i} u_{(i+1)}\right)=V\left(Q_{m}\right)-\left\{u_{((m+2 i+1) / 2)}, \quad v_{((m+2 i+1) / 2)}\right.$, $\left.w_{i}, z_{i}\right\}, \operatorname{LR}\left(u_{i} v_{i}\right)=V\left(Q_{m}\right)-\left\{v_{i+1}, v_{m}, w_{i+1}, z_{i+1}\right\}, \operatorname{LR}$ $\left(v_{i} w_{i}\right)=V\left(Q_{m}\right)-\left\{u_{i+1}, v_{i+2}, z_{m-1}, z_{m}\right\}, \quad \operatorname{LR}\left(z_{i} z_{i+1}\right)$ $=V\left(Q_{m}\right)-\left\{u_{i+1}, v_{i+1}, w_{((m+2 i-1) / 2)}, z_{((m+2 i-1) / 2)}\right\}$, LR $\left(w_{i} z_{i}\right)=V\left(Q_{m}\right)-\left\{v_{m}, w_{i+1}, w_{m}, v_{((m+2 i-1) / 2)}\right\}$, and $\left|\operatorname{LR}\left(e_{i}\right)\right|=4 m-4$, where $1 \leq i \leq m$.

Lemma 8. Let $Q_{m}$ with $m \geq 6$ be a network of convex polytope, where $m \cong 0(\bmod 2)$. Then, for $1 \leq i \leq m$,

(a) $\left|\operatorname{LR}\left(e_{i}\right)\right|=\left|\operatorname{LR}\left(v_{i} w_{i}\right)\right|=\left|\operatorname{LR}\left(w_{i} v_{i+1}\right)\right|=2 m+4, \quad \mid L R$ $\left(e_{i}^{\prime}\right)|=| L R\left(u_{i} u_{i+1}\right)|=| L R\left(u_{i} v_{i}\right)|=| L R\left(z_{i} z_{i+1}\right) \mid=$ $\left|L R\left(w_{i} z_{i}\right)\right|=4 m-4$, and $\left|\cup_{i=1}^{m} L R\left(e_{i}\right)\right|=4 m$

(b) $\left|L R\left(e_{i}^{\prime}\right) \cap \cup_{i=1}^{m} L R\left(e_{i}\right)\right| \geq\left|L R\left(e_{i}^{\prime}\right)\right|, \forall x \in E\left(Q_{m}\right)$

Proof. Assuming that $u_{i}, v_{i}, w_{i}$, and $z_{i}$ are inner, middle, and outer vertices, respectively, for $1 \leq i \leq m$ and $m+1 \cong$ $1(\bmod m)$, we have

(a) $\operatorname{LR}\left(v_{i} w_{i}\right)=V\left(Q_{m}\right)-\left\{u_{i+1}, u_{i+2}, u_{i+3}, \ldots, u_{((m+2 i) / 2)}\right.$, $v_{i+3}, v_{i+4}, \ldots, v_{((m+2 i) / 2)}, \quad w_{((m+2 i) / 2)}, w_{((m+2 i) / 2)+1}$, $w_{((m+2 i) / 2)+2}, \ldots, w_{m-2}, \quad z_{((m+2 i) / 2)}, z_{((m+2 i+3) / 2)}$, 
$\left.z_{((m+2 i)+5 / 2)}, \ldots, z_{m}\right\}$, and $\operatorname{LR}\left(w_{i} v_{i+1}\right)=V\left(Q_{n}\right)-$ $\left\{u_{i}, u_{((m+2 i+2) / 2)}, u_{((m+2 i+3) / 2)}, \ldots, u_{m}, \quad v_{((m+2 i+2) / 2)}\right.$, $v_{((m+2 i+2) / 2)+1}, v_{((m+2 i+2) / 2)+2}, \ldots, v_{m}, \quad w_{((m+2 i+2) / 2)}$, $\left.z_{i+1}, z_{i+2}, \ldots, z_{((m+2 i+2) / 2)}\right\}$; therefore, $\left|\operatorname{LR}\left(e_{i}\right)\right|=$ $2 m+4$, and also $\left|\cup_{i=1}^{m} \operatorname{LR}\left(e_{i}\right)\right|=\left|V\left(Q_{m}\right)\right|=4 m$. Moreover, $\operatorname{LR}\left(u_{i} u_{i+1}\right)=V\left(Q_{m}\right)-\left\{\left\{w_{i}, w_{((m+2 i) / 2)}\right.\right.$, $\left.z_{i}, z_{((m+2 i) / 2)}\right\}, \quad \operatorname{LR}\left(u_{i} v_{i}\right)=V\left(Q_{m}\right)-\left\{v_{i+1}, v_{m}, w_{i+1}\right.$, $\left.w_{m-1}\right\} \operatorname{LR}\left(z_{i} z_{i+1}\right)=V\left(Q_{m}\right)-\left\{u_{i+1}, v_{i+1}, v_{((m+2 i+2) / 2)}\right.$, $\left.u_{((m+2 i+2) / 2)}\right\}$, and $\operatorname{LR}\left(w_{i} z_{i}\right)=V\left(Q_{m}\right)-\left\{v_{i+2}, v_{m}\right.$, $\left.w_{i+1}, w_{m}\right\}$; therefore, $\left|\operatorname{LR}\left(e_{i}^{\prime}\right)\right|=4 m-4$.

(b) Since $\cup_{i=1}^{m} \operatorname{LR}\left(e_{i}\right)=V\left(Q_{m}\right)$, therefore $\mid \operatorname{LR}\left(e_{i}^{\prime}\right) \cap$ $\cup_{i=1}^{m} \operatorname{LR}\left(e_{i}\right)|=| \operatorname{LR}\left(e_{i}^{\prime}\right)|\geq| \operatorname{LR}\left(e_{i}\right) \mid$.

Theorem 9. Let $Q_{m}$ with $m \geq 5$ be a network of convex polytope, where $m \cong 1(\bmod 2)$. Then,

$$
\operatorname{dim}_{f l}\left(Q_{m}\right)=\frac{m}{m-1} \text {. }
$$

Proof. For $m=5$, the LRN sets are as follows:

$$
\begin{aligned}
& \operatorname{LR}_{1}=\operatorname{LR}\left(u_{1} u_{2}\right)=V\left(Q_{5}\right)-\left\{u_{4}, v_{4}, w_{1}, z_{1}\right\}, \\
& \mathrm{LR}_{2}=\operatorname{LR}\left(u_{2} u_{3}\right)=V\left(Q_{5}\right)-\left\{u_{5}, v_{5}, w_{2}, z_{2}\right\} \text {, } \\
& \mathrm{LR}_{3}=\operatorname{LR}\left(u_{3} u_{4}\right)=V\left(Q_{5}\right)-\left\{u_{1}, v_{1}, w_{3}, z_{3}\right\} \text {, } \\
& \mathrm{LR}_{4}=\operatorname{LR}\left(u_{4} u_{5}\right)=V\left(Q_{5}\right)-\left\{u_{2}, v_{2}, w_{4}, z_{4}\right\}, \\
& \mathrm{LR}_{5}=\operatorname{LR}\left(u_{5} u_{1}\right)=V\left(Q_{5}\right)-\left\{u_{3}, v_{3}, w_{5}, z_{5}\right\} \text {, } \\
& \mathrm{LR}_{6}=\operatorname{LR}\left(u_{1} v_{1}\right)=V\left(Q_{5}\right)-\left\{v_{2}, v_{5}, w_{2}, w_{4}\right\} \text {, } \\
& \mathrm{LR}_{7}=\operatorname{LR}\left(u_{2} v_{2}\right)=V\left(Q_{5}\right)-\left\{v_{3}, v_{1}, w_{3}, w_{5}\right\} \text {, } \\
& \mathrm{LR}_{8}=\operatorname{LR}\left(u_{3} v_{3}\right)=V\left(Q_{5}\right)-\left\{v_{4}, v_{2}, w_{4}, w_{1}\right\} \text {, } \\
& \operatorname{LR}_{9}=\operatorname{LR}\left(u_{4} v_{4}\right)=V\left(Q_{5}\right)-\left\{v_{5}, v_{3}, w_{5}, w_{2}\right\}, \\
& \mathrm{LR}_{10}=\operatorname{LR}\left(u_{5} v_{5}\right)=V\left(Q_{5}\right)-\left\{v_{1}, v_{4}, w_{1}, w_{3}\right\} \text {, } \\
& \operatorname{LR}_{11}=\operatorname{LR}\left(v_{1} w_{1}\right)=V\left(Q_{5}\right)-\left\{u_{2}, u_{3}, z_{4}, z_{5}\right\} \text {, } \\
& \operatorname{LR}_{12}=\operatorname{LR}\left(v_{2} w_{2}\right)=V\left(Q_{5}\right)-\left\{u_{3}, u_{4}, z_{5}, z_{1}\right\} \text {, } \\
& \operatorname{LR}_{13}=\operatorname{LR}\left(v_{3} w_{3}\right)=V\left(Q_{5}\right)-\left\{u_{4}, u_{5}, z_{1}, z_{2}\right\} \text {, } \\
& \operatorname{LR}_{14}=\operatorname{LR}\left(v_{4} w_{4}\right)=V\left(Q_{5}\right)-\left\{u_{5}, u_{1}, z_{2}, z_{3}\right\} \text {, } \\
& \operatorname{LR}_{15}=\operatorname{LR}\left(v_{5} w_{5}\right)=V\left(Q_{5}\right)-\left\{u_{1}, u_{2}, z_{3}, z_{4}\right\} \text {, } \\
& \operatorname{LR}_{16}=\operatorname{LR}\left(w_{1} v_{2}\right)=V\left(Q_{5}\right)-\left\{u_{1}, u_{5}, z_{2}, z_{3}\right\} \text {, } \\
& \operatorname{LR}_{17}=\operatorname{LR}\left(w_{2} v_{3}\right)=V\left(Q_{5}\right)-\left\{u_{2}, u_{1}, z_{3}, z_{4}\right\} \text {, } \\
& \operatorname{LR}_{18}=\operatorname{LR}\left(w_{3} v_{4}\right)=V\left(Q_{5}\right)-\left\{u_{3}, u_{2}, z_{4}, z_{5}\right\} \text {, } \\
& \operatorname{LR}_{19}=\operatorname{LR}\left(w_{4} v_{5}\right)=V\left(Q_{5}\right)-\left\{u_{4}, u_{3}, z_{5}, z_{1}\right\} \text {, } \\
& \operatorname{LR}_{20}=\operatorname{LR}\left(w_{5} v_{1}\right)=V\left(Q_{5}\right)-\left\{u_{5}, u_{4}, z_{1}, z_{2}\right\} \text {, } \\
& \operatorname{LR}_{21}=\operatorname{LR}\left(w_{1} z_{1}\right)=V\left(Q_{5}\right)-\left\{v_{2}, v_{5}, w_{2}, w_{5}\right\} \text {, } \\
& \operatorname{LR}_{22}=\operatorname{LR}\left(w_{2} z_{2}\right)=V\left(Q_{5}\right)-\left\{v_{3}, v_{1}, w_{3}, w_{1}\right\} \text {, } \\
& \operatorname{LR}_{23}=\operatorname{LR}\left(w_{3} z_{3}\right)=V\left(Q_{5}\right)-\left\{v_{4}, v_{2}, w_{4}, w_{2}\right\} \text {, } \\
& \mathrm{LR}_{24}=\operatorname{LR}\left(w_{4} z_{4}\right)=V\left(Q_{5}\right)-\left\{v_{5}, v_{3}, w_{5}, w_{3}\right\} \text {, } \\
& \operatorname{LR}_{25}=\operatorname{LR}\left(w_{5} z_{5}\right)=V\left(Q_{5}\right)-\left\{v_{1}, v_{4}, w_{1}, w_{4}\right\}, \\
& \mathrm{LR}_{26}=\operatorname{LR}\left(z_{1} z_{2}\right)=V\left(Q_{5}\right)-\left\{u_{2}, v_{2}, w_{4}, z_{4}\right\} \text {, } \\
& \operatorname{LR}_{27}=\operatorname{LR}\left(z_{2} z_{3}\right)=V\left(Q_{5}\right)-\left\{v_{3}, u_{3}, w_{5}, z_{5}\right\} \text {, } \\
& \operatorname{LR}_{28}=\operatorname{LR}\left(z_{3} z_{4}\right)=V\left(Q_{5}\right)-\left\{v_{4}, u_{4}, w_{1}, z_{1}\right\} \text {, } \\
& \mathrm{LR}_{29}=\operatorname{LR}\left(z_{4} z_{5}\right)=V\left(Q_{5}\right)-\left\{v_{5}, u_{5}, w_{2}, z_{2}\right\} \text {, } \\
& \operatorname{LR}_{30}=\operatorname{LR}\left(z_{5} z_{1}\right)=V\left(Q_{5}\right)-\left\{v_{1}, u_{1}, w_{3}, z_{3}\right\} \text {. }
\end{aligned}
$$

Each $\left|\operatorname{LR}\left(e_{i}\right)\right|=16$, where $1 \leq i \leq 30$. Furthermore, $\left|\cup_{i=1}^{30} \operatorname{LR}\left(e_{i}\right)\right|=20$. Therefore, by Corollary 1 ,

$$
\operatorname{dim}_{f l}\left(Q_{5}\right)=\frac{5}{4}
$$

For $m>5$, by Lemma $7, \quad\left|\operatorname{LR}\left(e_{i}\right)\right|=\left|\operatorname{LR}\left(u_{i} u_{i+1}\right)\right|$ $=\left|\operatorname{LR}\left(z_{i} z_{i+1}\right)\right|=\left|\operatorname{LR}\left(u_{i} v_{i}\right)\right|=\left|\operatorname{LR}\left(v_{i} w_{i}\right)\right|=\left|\operatorname{LR}\left(w_{i} z_{i}\right)\right|=4 m$ -4 and $\left|\cup_{i=1}^{m} \operatorname{LR}\left(e_{i}\right)\right|=4 m$, where $1 \leq i \leq m$. Hence, from Corollary 1 , we have

$$
\operatorname{dim}_{f l}\left(Q_{m}\right)=\frac{m}{m-1} .
$$

Theorem 10. Let $Q_{m}$ with $m \geq 6$ be a network of convex polytope, where $m \cong 0(\bmod 2)$.

$$
\frac{m}{m-1} \leq \operatorname{dim}_{f l}\left(Q_{m}\right) \leq \frac{2 m}{m+2}
$$

Proof. To prove the result, we have following cases:

Case 1. For $m=6$, the LRN sets are as follows:

$$
\begin{aligned}
& \mathrm{LR}_{1}=\operatorname{LR}\left(u_{1} u_{2}\right)=V\left(Q_{6}\right)-\left\{w_{1}, w_{4}, z_{1}, z_{4}\right\}, \\
& \mathrm{LR}_{2}=\operatorname{LR}\left(u_{2} u_{3}\right)=V\left(Q_{6}\right)-\left\{w_{2}, w_{5}, z_{2}, z_{5}\right\} \text {, } \\
& \mathrm{LR}_{3}=\operatorname{LR}\left(u_{3} u_{4}\right)=V\left(Q_{6}\right)-\left\{w_{3}, w_{6}, z_{3}, z_{6}\right\} \text {, } \\
& \mathrm{LR}_{4}=\operatorname{LR}\left(u_{4} u_{5}\right)=V\left(Q_{6}\right)-\left\{w_{4}, w_{1}, z_{4}, z_{1}\right\} \text {, } \\
& \mathrm{LR}_{5}=\operatorname{LR}\left(u_{5} u_{6}\right)=V\left(Q_{6}\right)-\left\{w_{5}, w_{2}, z_{5}, z_{2}\right\} \text {, } \\
& \mathrm{LR}_{6}=\operatorname{LR}\left(u_{6} u_{1}\right)=V\left(Q_{6}\right)-\left\{w_{6}, w_{3}, z_{6}, z_{3}\right\} \text {, } \\
& \mathrm{LR}_{7}=\operatorname{LR}\left(u_{1} v_{1}\right)=V\left(Q_{6}\right)-\left\{v_{2}, v_{6}, w_{2}, w_{5}\right\} \text {, } \\
& \mathrm{LR}_{8}=\operatorname{LR}\left(u_{2} v_{2}\right)=V\left(Q_{6}\right)-\left\{v_{3}, v_{1}, w_{3}, w_{6}\right\}, \\
& \mathrm{LR}_{9}=\mathrm{LR}\left(u_{3} v_{3}\right)=V\left(Q_{6}\right)-\left\{v_{4}, v_{2}, w_{4}, w_{1}\right\}, \\
& \operatorname{LR}_{10}=\operatorname{LR}\left(u_{4} v_{4}\right)=V\left(Q_{6}\right)-\left\{v_{5}, v_{3}, w_{5}, w_{2}\right\} \text {, } \\
& \operatorname{LR}_{11}=\operatorname{LR}\left(u_{5} v_{5}\right)=V\left(Q_{6}\right)-\left\{v_{6}, v_{4}, w_{6}, w_{3}\right\} \text {, } \\
& \mathrm{LR}_{12}=\operatorname{LR}\left(u_{6} v_{6}\right)=V\left(Q_{6}\right)-\left\{v_{1}, v_{5}, w_{1}, w_{4}\right\} \text {, } \\
& \mathrm{LR}_{13}=\operatorname{LR}\left(w_{1} z_{1}\right)=V\left(Q_{6}\right)-\left\{v_{3}, v_{6}, w_{2}, w_{6}\right\} \text {, } \\
& \operatorname{LR}_{14}=\operatorname{LR}\left(w_{2} z_{2}\right)=V\left(Q_{6}\right)-\left\{v_{4}, v_{1}, w_{3}, w_{1}\right\}, \\
& \operatorname{LR}_{15}=\operatorname{LR}\left(w_{3} z_{3}\right)=V\left(Q_{6}\right)-\left\{v_{5}, v_{2}, w_{4}, w_{2}\right\}, \\
& \mathrm{LR}_{16}=\operatorname{LR}\left(w_{4} z_{4}\right)=V\left(Q_{6}\right)-\left\{v_{6}, v_{3}, w_{5}, w_{3}\right\}, \\
& \mathrm{LR}_{17}=\operatorname{LR}\left(w_{5} z_{5}\right)=V\left(Q_{6}\right)-\left\{v_{1}, v_{4}, w_{6}, w_{4}\right\} \text {, } \\
& \operatorname{LR}_{18}=\operatorname{LR}\left(w_{6} z_{6}\right)=V\left(Q_{6}\right)-\left\{v_{2}, v_{3}, w_{1}, w_{5}\right\} \text {, } \\
& \mathrm{LR}_{19}=\operatorname{LR}\left(z_{1} z_{2}\right)=V\left(Q_{6}\right)-\left\{u_{2}, u_{5}, v_{2}, v_{5}\right\} \text {, } \\
& \mathrm{LR}_{20}=\operatorname{LR}\left(z_{2} z_{3}\right)=V\left(Q_{6}\right)-\left\{u_{3}, u_{6}, v_{3}, v_{6}\right\} \text {, } \\
& \operatorname{LR}_{21}=\operatorname{LR}\left(z_{3} z_{4}\right)=V\left(Q_{6}\right)-\left\{u_{4}, u_{1}, v_{4}, v_{1}\right\} \text {, } \\
& \mathrm{LR}_{22}=\operatorname{LR}\left(z_{4} z_{5}\right)=V\left(Q_{6}\right)-\left\{u_{5}, u_{2}, v_{5}, v_{2}\right\} \text {, } \\
& \operatorname{LR}_{23}=\operatorname{LR}\left(z_{5} z_{6}\right)=V\left(Q_{6}\right)-\left\{u_{6}, u_{3}, v_{6}, v_{3}\right\}, \\
& \mathrm{LR}_{24}=\operatorname{LR}\left(z_{6} z_{1}\right)=V\left(Q_{6}\right)-\left\{u_{1}, u_{4}, v_{1}, v_{4}\right\} \text {, }
\end{aligned}
$$


TABLE 7: Limiting values of LFMDs of convex polytopes networks.

\begin{tabular}{lccc}
\hline$m \cong$ & LFMDs & Limiting LFMDs for $m \longrightarrow \infty$ & Comment \\
\hline $1(\bmod 2)$ & $1<\operatorname{dim}_{f l}\left(R_{m}\right) \leq(2 m /(m+1))$ & $1<\operatorname{dim}_{f l}\left(R_{m}\right) \leq 2$ & Bounded \\
$1(\bmod 2)$ & $1<\operatorname{dim}_{f l}\left(A_{m}\right) \leq(2 m /(m+1))$ & $1<\operatorname{dim}_{f l}\left(S_{m}\right) \leq 2$ & Bounded \\
$0(\bmod 2)$ & $(m /(m-1)) \leq \operatorname{dim}_{f l}\left(A_{m}\right) \leq(2 m /(m+2))$ & $1<\operatorname{dim}_{f l}\left(S_{m}\right) \leq 2$ & Bounded \\
$1(\bmod 2)$ & $1 \leq \operatorname{dim}_{f l}\left(S_{m}\right) \leq(2 m /(m+1))$ & $1<\operatorname{dim}_{f l}\left(S_{m}\right) \leq 2$ & Bounded \\
$0(\bmod 2)$ & $(m /(m-1)) \leq \operatorname{dim}_{f l}\left(Q_{m}\right) \leq(2 m /(m+2))$ & $1<\operatorname{dim}_{f l}\left(S_{m}\right) \leq 2$ & Bounded \\
\hline
\end{tabular}

TABLE 8: Constant and bounded values of LFMD of convex polytopes networks.

\begin{tabular}{ll}
\hline$m \cong$ & $1<\operatorname{dim}$ \\
\hline $0(\bmod 2)$ & $1<\operatorname{dim}$ \\
$0(\bmod 2)$ & \\
\hline & \\
$L_{25}=\operatorname{LR}\left(v_{1} w_{1}\right)=V\left(Q_{6}\right)-\left\{u_{2}, u_{3}, u_{4}, v_{2}, v_{4}, z_{4}, z_{5}, z_{6}\right\}$, \\
$\operatorname{LR}_{26}=\operatorname{LR}\left(v_{2} w_{2}\right)=V\left(Q_{6}\right)-\left\{u_{3}, u_{4}, u_{5}, v_{3}, v_{5}, z_{5}, z_{6}, z_{1}\right\}$, \\
$\mathrm{LR}_{27}=\operatorname{LR}\left(v_{3} w_{3}\right)=V\left(Q_{6}\right)-\left\{u_{4}, u_{5}, u_{6}, v_{4}, v_{6}, z_{6}, z_{1}, z_{2}\right\}$, \\
$\mathrm{LR}_{28}=\operatorname{LR}\left(v_{4} w_{4}\right)=V\left(Q_{6}\right)-\left\{u_{5}, u_{6}, u_{1}, v_{5}, v_{1}, z_{1}, z_{2}, z_{3}\right\}$, \\
$\mathrm{LR}_{29}=\operatorname{LR}\left(v_{5} w_{5}\right)=V\left(Q_{6}\right)-\left\{u_{6}, u_{1}, u_{2}, v_{6}, v_{2}, z_{2}, z_{3}, z_{4}\right\}$, \\
$\mathrm{LR}_{30}=\operatorname{LR}\left(v_{6} w_{6}\right)=V\left(Q_{6}\right)-\left\{u_{1}, u_{2}, u_{3}, v_{1}, v_{3}, z_{3}, z_{4}, z_{5}\right\}$, \\
$\mathrm{LR}_{31}=\operatorname{LR}\left(w_{1} v_{2}\right)=V\left(Q_{6}\right)-\left\{u_{1}, u_{5}, u_{6}, v_{5}, w_{4}, z_{2}, z_{3}, z_{4}\right\}$, \\
$\mathrm{LR}_{32}=\operatorname{LR}\left(w_{2} v_{3}\right)=V\left(Q_{6}\right)-\left\{u_{2}, u_{6}, u_{1}, v_{6}, w_{5}, z_{3}, z_{4}, z_{5}\right\}$, \\
$\mathrm{LR}_{33}=\operatorname{LR}\left(w_{3} v_{4}\right)=V\left(Q_{6}\right)-\left\{u_{3}, u_{1}, u_{2}, v_{1}, w_{6}, z_{4}, z_{5}, z_{6}\right\}$, \\
$\operatorname{LR}_{34}=\operatorname{LR}\left(w_{4} v_{5}\right)=V\left(Q_{6}\right)-\left\{u_{4}, u_{2}, u_{3}, v_{2}, w_{1}, z_{5}, z_{6}, z_{1}\right\}$, \\
$\operatorname{LR}_{35}=\operatorname{LR}\left(w_{5} v_{6}\right)=V\left(Q_{6}\right)-\left\{u_{5}, u_{3}, u_{4}, v_{3}, w_{2}, z_{6}, z_{1}, z_{2}\right\}$, \\
$\operatorname{LR}_{36}=\operatorname{LR}\left(w_{6} v_{1}\right)=V\left(Q_{6}\right)-\left\{u_{5}, u_{4}, u_{5}, v_{4}, w_{3}, z_{1}, z_{2}, z_{3}\right\}$.
\end{tabular}

For $1 \leq i \leq 6,\left|\operatorname{LR}\left(e_{i}\right)\right|=\left|\operatorname{LR}\left(v_{i} w_{i}\right)\right|=\left|\operatorname{LR}\left(w_{i} v_{i+1}\right)\right|=16$ and $\left|\operatorname{LR}\left(e_{i}\right)\right| \leq|\operatorname{LR}(x)|, \forall x \in E\left(Q_{6}\right)$. Furthermore, $\left|\cup_{i=1}^{6} \operatorname{LR}\left(e_{i}\right)\right|=24$. Thus, there exists an upper LRF $\phi: V\left(Q_{6}\right) \longrightarrow[0,1]$ defined as $\phi(v)=(1 / 16), \forall v \in$ $V\left(Q_{6}\right)$. Consequently, by Theorem $1, \operatorname{dim}_{f l}\left(Q_{6}\right) \leq$ (3/2). Similarly, for $1 \leq i \leq 6,\left|\operatorname{LR}\left(e_{i}^{\prime}\right)\right|=\left|\operatorname{LR}\left(u_{i} v_{i}\right)\right|=$ $\left|\operatorname{LR}\left(w_{i} z_{i}\right)\right|=\left|\operatorname{LR}\left(z_{i} z_{i+1}\right)\right|=20$ and $\left|\operatorname{LR}\left(e_{i}^{\prime}\right)\right| \geq \mid \operatorname{LR}$ $(x) \mid, \forall x \in E\left(Q_{6}\right)$. Thus, there exists a LRF $\phi^{\prime}: V$ $\left(Q_{6}\right) \longrightarrow[0,1]$ defined as $\phi^{\prime}(v)=(1 / 20)$ which is a lower LRF $\forall v \in V\left(Q_{6}\right)$. Consequently, by Theorem 2 , $\operatorname{dim}_{f l}\left(Q_{6}\right) \geq(6 / 5)$. Hence, we have

$$
\frac{6}{5} \leq \operatorname{dim}_{f l}\left(Q_{6}\right) \leq \frac{3}{2}
$$

Case 2. For $m>6$, by Lemma 8, $\left|\operatorname{LR}\left(e_{i}\right)\right|=\left|\operatorname{LR}\left(v_{i} w_{i}\right)\right|=$ $\left|\operatorname{LR}\left(w_{i} v_{i+1}\right)\right|=2 m+4$ and $\left|\operatorname{LR}\left(e_{i}\right)\right| \leq|\operatorname{LR}(x)|, \forall x \in E$ $\left(Q_{m}\right)$. Furthermore, $\left|\operatorname{LR}\left(e_{i}^{\prime}\right)\right|=\left|\operatorname{LR}\left(u_{i} u_{i+1}\right)\right|,\left|\operatorname{LR}\left(u_{i} v_{i}\right)\right|$, $\left|\operatorname{LR}\left(z_{i} z_{i+1}\right)\right|$ with $\left|\operatorname{LR}\left(w_{i} z_{i}\right)\right|=4 m-4$ and $\left|\operatorname{LR}\left(e_{i}^{\prime}\right)\right|$ $\geq|\operatorname{LR}(x)|, \quad \forall x \in E\left(Q_{m}\right)$. Therefore, $\phi: V\left(Q_{m}\right) \longrightarrow$ $[0,1]$ defined by $\phi(v)=(1 /(2 m+4)), \forall v \in V\left(R_{m}\right)$ is an upper LRF and $\phi^{\prime}: V\left(Q_{m}\right) \longrightarrow[0,1]$ defined by $\phi^{\prime}(v)=(1 /(4 m-4)), \forall v \in V\left(Q_{m}\right)$ is a lower LRF. Therefore, by Theorems 1 and 2, we have

$$
\frac{m}{m-1} \leq \operatorname{dim}_{f l}\left(Q_{m}\right) \leq \frac{2 m}{m+2}
$$

\section{Conclusion}

In this article, we studied the boundedness of convex polytopes networks with the help of distance-based parameter that is called by local fractional metric dimension (LFMD). The lower bounds of convex polytopes networks are also improved from unity. The LFMD of the convex polytope $Q_{m}$, where $m \cong(1 \bmod 2)$ is $(m /(m-1))$. Furthermore, other families of convex polytopes such as $R_{m}, A_{m}, S_{m}$, and $Q_{m}$ attain both upper and lower bounds between 1 and 2, respectively, as their order approaches to $\infty$.

Now, we close our discussion with an open problem and characterize all classes of connected networks those remain bounded via LFMD when $m \longrightarrow \infty$.

Limiting values of convex polytopes networks are illustrated in Tables 7 and 8 .

\section{Data Availability}

All the data are included within this paper. However, the reader may contact the corresponding author for more details of the data.

\section{Conflicts of Interest}

The authors declare that there are no conflicts of interest regarding this article.

\section{References}

[1] P. J. Slater, "Leaves of trees," Congressus Numerantium, vol. 14 , pp. 549-559, 1975.

[2] F. Harary and R. Melter, "On the metric dimension of a graph,” Ars Combinatoria, vol. 2, pp. 19-195, 1976.

[3] M. R. Garey and D. S. Johnson, Computers and Intractibility, A Guide to the Theory of NP-Completeness, W. H. Freeman and Company, New York, NY, USA, 1979.

[4] R. A. Melter and I. Tomescu, "Metric bases in digital geometry," Computer Vision, Graphics, and Image Processing, vol. 25, no. 1, pp. 113-121, 1984. 
[5] G. Chartrand, L. Eroh, M. Johnson, and O. R. Oellermann, "Resolvability in graphs and the metric dimension of a graph," Discrete Applied Mathematics, vol. 105, 2000.

[6] M. Imran, M. K. Siddiqui, and R. Naeem, "On the metric dimension of generalized petersen multigraphs," IEEE Access, vol. 6, pp. 74328-74338, 2018.

[7] M. Imran, A. Q. Baig, M. K. Shafiq, and I. Tomescu, "On metric dimension of generalized Petersen graphs $\mathrm{P}(\mathrm{n} ; 3)$," ARS Combinatoria, vol. 117, pp. 113-130, 2014.

[8] F. Okamoto, B. Phinezy, and P. Zhang, "The local metric dimension of a graph," Mathematica Bohemica, vol. 135, no. 3 , pp. 239-255, 2010.

[9] A. Sebo and E. Tannier, "On metric generators of graphs," Mathematics of Operations Research, vol. 29, no. 2, pp. 383-393, 2004.

[10] I. G. Yero, A. Estrada-Moreno, and J. A. RodríguezVelázquez, "Computing the $\mathrm{k}$-metric dimension of graphs," Applied Mathematics and Computation, vol. 300, pp. 60-69, 2017.

[11] Y.-M. Chu, M. F. Nadeem, M. Azeem, and M. K. Siddiqui, "On sharp bounds on partition dimension of convex polytopes," IEEE Access, vol. 8, pp. 224781-224790, 2020.

[12] X. Zuo, A. Ali, G. Ali, M. K. Siddiqui, M. Tariq Rahim, and A. Asare-Tuah, "'On constant metric dimension of some generalized convex polytopes," Journal of Mathematics, vol. 2021, Article ID 6919858, 7 pages, 2021.

[13] H. Pan, M. Ali, G. Ali, M. T. Rahim, and X. Yang, "On the families of graphs with unbounded metric dimension," IEEE Access, vol. 7, pp. 165060-165064, 2019.

[14] M. Perc, J. Gómez-Gardeñes, A. Szolnoki, L. M. Floría, and Y. Moreno, "Evolutionary dynamics of group interactions on structured populations: a review," Journal of The Royal Society Interface, vol. 10, no. 80, Article ID 20120997, 2013.

[15] M. Perc and A. Szolnoki, "Coevolutionary games-A mini review," Biosystems, vol. 99, no. 2, pp. 109-125, 2010.

[16] I. González Yero, M. Jakovac, D. Kuziak, and A. Taranenko, "The partition dimension of strong product graphs and Cartesian product graphs," Discrete Mathematics, vol. 331, pp. 43-52, 2014.

[17] J. Currie and O. R. Oellermann, "The metric dimension and metric independence of a graph," Journal of Combinatorial Mathematics and Combinatorial Computing, vol. 39, pp. 157-167, 2001.

[18] M. Fehr, S. Gosselin, and O. R. Oellermann, "The metric dimension of Cayley digraphs," Discrete Mathematics, vol. 306, no. 1, pp. 31-41, 2006.

[19] S. Arumugam and V. Mathew, "The fractional metric dimension of graphs," Discrete Mathematics, vol. 312, no. 9, 2012.

[20] A. H. Alkhaldi, M. K. Aslam, M. Javaid, and A. M. Alanazi, "Bounds of fractional metric dimension and applications with grid-Related networks," Mathematics, vol. 9, no. 12, p. 1383, 2021.

[21] J.-B. Liu, A. Kashif, T. Rashid, and M. Javaid, "Fractional metric dimension of generalized Jahangir graph," Mathematics, vol. 7, pp. 1-10, 2019.

[22] A. Daniel and S. WidoSaputro, "Fractional metric dimension of tree and unicyclic graph procedia," Computer Science, vol. 74, pp. 47-52, 2015.

[23] M. Javaid, K. Aslam, A. M. Alanazi, and M. Aljohani, "Characterization of (Molecular) graphs with fractional metric dimension as unity," Journal of Chemistry, vol. 2021, Article ID 9910572, 12 pages, 2021.
[24] M. Raza, D. Alrowalili, M. Javaid, and K. Shabbir, "Computing bounds of fractional metric dimension of metal organic graphs," Journal of Chemistry, vol. 202112 pages, 2021.

[25] M. Feng, L. Benjian, and W. Kaishun, "On the fractional metric dimension of graphs," Discrete Mathematics-DM, vol. 170, 2011.

[26] S. Aisyah, M. Utoyo, and L. Susilowati, "On the local fractional metric dimension of corona product graphs," IOP Conf., Earth Environ. Sci, Hungarica, vol. 243, pp. 1-4, 2019.

[27] M. Javaid, M. Raza, P. Kumam, and J.-B. Liu, "Sharp bounds of local fractional metric dimesion of connected networks," IEEE Access, vol. 8, pp. 172329-172342, 202.

[28] M. Javaid, H. Zafar, Q. Zhu, and A. M. Alanazi, "Improved lower bound of LFMD with applications of prism-related networks," Mathematical Problems in Engineering, vol. 2021, Article ID 9950310, 9 pages, 2021.

[29] M. Baca, "Labelings of two classes of convex polytopes," Utilitas Mathematica, vol. 34, pp. 24-31, 1988.

[30] D. B. West, Introduction to Graph Theory, Prentice-Hall, Hoboken, NJ, USA, 2 edition, 2011. 